\title{
Messung der Diffusionsverluste von radiogenen und spallogenen Edelgasen in Steinmeteoriten II
}

\author{
H. Hintenberger, L. Schultz und H. Wänke \\ Max-Planck-Institut für Chemie, (Otto-Hahn-Institut), Mainz \\ (Z. Naturforschg. 21 a, 1147-1159 [1966] ; eingegangen am 7. März 1966) \\ Professor Dr. W. Gentner zum 60. Geburtstag gewidmet
}

\begin{abstract}
Rare gas measurements (helium and neon) were carried out on single mineral components of 10 bronzite-chondrites, 10 hypersthene-chondrites and 1 amphoterite. Diffusion losses of ${ }^{3} \mathrm{He}$ were detected in pyroxene on most meteorites studied. The helium content of olivine was found to be far less effected by diffusion compared to pyroxene. A loss of spallogenic helium is always connected with a loss of radiogenic helium.

For most of the bronzite-chondrites the loss of radiogenic helium occured only during the time of cosmic ray exposure due to a more intensive heating caused by orbits closer to the sun. Bronzitechondrites containing high amounts of primordial gases showed equal relative losses of spallogenic and radiogenic helium each. The reason for this unexpected results is not clear, but it might be that it results from a grain-size-effect and perhaps also holds true for all other meteorites as far as diffusion loss in space due to solar heating is concerned.

Many of hypersthene-chondrites suffered inside their parent bodies considerable losses of radiogenic helium before their exposure to the cosmic rays. From the radiogenic helium content in pyroxene we calculated an age of $560 \cdot 10^{6}$ years for five of these meteorites, but we are not quite sure that this is a true age.

It could be proved that the highly varying ratio of ${ }^{3} \mathrm{He} /{ }^{21} \mathrm{Ne}$ in chondrites cannot be attributed to diffusion losses. Variations up to a factor of 4 were confirmed.

The content of ${ }^{3} \mathrm{He}$ in the metal phase of most meteorites investigated was found to be too low up to a factor of 2. A tritium loss in space is suggested as explanation of these deficiencies.
\end{abstract}

In den letzten fünf Jahren wurden Edelgasanalysen an einer sehr großen Zahl von Steinmeteoriten veröffentlicht. Die meisten dieser Messungen stammen aus den Laboratorien von Heidelberg ${ }^{1-3}$ und Mainz ${ }^{4-6}$. Unter Berücksichtigung unveröffentlichter Ergebnisse können wir sagen, daß von etwa der Hälfte aller klassifizierten Chondrite, deren Fall beobachtet wurde, Edelgasanalysen durchgeführt worden sind. Aus diesen Messungen konnten Werte für die radiogenen Alter sowie für die $\mathrm{Be}$ strahlungsalter der Meteorite berechnet werden. Daraus ergeben sich zahlreiche wertvolle Folgerungen. Es traten jedoch eine Reihe von Fragen auf, die teilweise bis heute unbeantwortet blieben. So wurden Diffusionsverluste von radiogenem Helium festgestellt, deren Zeitpunkt und Ursache bis jetzt ziemlich ungeklärt blieb. Keine der verschiedenen Нypothesen 2, 4, 6, 7 zur Erklärung der gefundenen starken Variationen des ${ }^{3} \mathrm{He} /{ }^{21} \mathrm{Ne}-$ Verhältnisses konnte eindeutig bewiesen, kaum eine auch wirklich widerlegt

1 J. Zähringer, Z. Naturforschg. 17 a, 460 [1962].

2 T. Kirsten, D. Krankowsky u. J. Zähringer, Geochim. Cosmochim. Acta 27, 13 [1963].

3 J. Z̈̈HRINGER, Vorabdruck 1964; erscheint in Meteoritika.

4 H. Hintenberger, H. König u. H. Wänke, Z. Naturforschg. 17 a, 1092 [1962ך. werden. Auch hier kann es durch Diffusionsverluste - vom leichteren Helium geht hierbei natürlich mehr verloren - zu Verschiebungen $\operatorname{des}{ }^{3} \mathrm{He} /{ }^{21} \mathrm{Ne}-V e r-$ hältnisses kommen.

Vor einiger Zeit haben wir Versuche mit dem Ziel begonnen, zumindest einige der mit Diffusionsverlusten zusammenhängenden Fragen zu klären. Wir sind hierbei von der Tatsache ausgegangen, daß die Chondrite aus mehreren recht unterschiedlichen Mineralkomponenten aufgebaut sind. Es war zu vermuten, daß diese Mineralkomponenten verschiedene Diffusionseigenschaften aufweisen würden.

Wir haben ein Verfahren entwickelt, das ohne großen Aufwand erlaubt, den Edelgasgehalt der einzelnen Mineralkomponenten getrennt zu bestimmen. Vor allem kam es uns darauf an, daß stets tatsächlich die gesamte Probe analysiert werden konnte und keine Fraktionierung nach der Korngröße auftrat. Gerade die feinsten Körner sind in erster Linie für die Edelgasverluste verantwortlich.

5 H. Hintenberger, H. König, L. Schultz u. H. Wänke, Z. Naturforschg. 19 a, 327 [1964].

6 H. Hintenberger, H. König, L. Schultz u. H. Wänke, Z. Naturforschg. 20 a, 983 [1965].

7 H. Stauffer, J. Geophys. Res. 67, 2023 [1962]. 
Zur Lösung der gestellten Aufgabe bedienten wir uns eines Verfahrens, das auf der unterschiedlichen Resistenz der einzelnen Mineralkomponenten gegenüber verschiedenen chemischen Reagenzien beruht (VILCSEK u. W ÄNKE $^{8}$ ). Auch konnten in einer ersten Arbeit ${ }^{9}$ bereits Ergebnisse solcher Messungen an fünf Meteoriten mitgeteilt werden.

\section{Meßergebnisse}

Nunmehr liegen Messungen an 21 Steinmeteoriten vor, und zwar an 10 Bronzit-Chondriten, 10 Hypersthen-Chondriten und einem amphoteritischen Chondriten. Die Ergebnisse sind in Tab. 1 zusammengefaßt. Die in den einzelnen Reagenzien gelösten Mineralbestandteile sowie deren Menge wurden durch chemische Analysen der in Lösung gegangenen Elemente bestimmt. Die gepulverten Meteoritproben wurden nacheinander mit folgenden Lösungsmitteln behandelt ${ }^{8}$ :

1. $\mathrm{CuCl}_{2}$ zur Lösung des Nickeleisens,

2. Bromwasser zur Lösung des Troilits und

3. Salzsäure zur Lösung des Olivins.

Der unlösliche Rückstand enthält neben Pyroxen (Hauptmenge) auch noch die Feldspäte. Spurenminerale wie Apatit bzw. eventuell vorhandener Schreibersit blieben unberücksichtigt. (Beide Minerale würden in der Bromphase in Lösung gehen.) Die Edelgase dieser einzelnen Fraktionen wurden getrennt gemessen ${ }^{9}$.

Beim Vergleich der Summenwerte der Edelgaskonzentrationen mit den Literaturwerten ist der Umstand zu berücksichtigen, daß auch die Addition aller Mineralien selten $100 \%$ ergibt.

Der Fehlbetrag ist auf Verluste von Meteoritpulver beim mehrmaligen Umspülen der Probe im Laufe der Extraktionsschritte zurückzuführen.

Zur Berechnung des radiogenen Heliums wurde

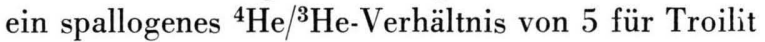
und Silikate angenommen. Dieser Wert entstammt Untersuchungen von Heymans ${ }^{10}$. Für das metallische Nickeleisen blieben wir bei einem Verhältnis von 4, da wir annehmen, daß es sich bei dem überschüssigen ${ }^{4} \mathrm{He}$ - gegenüber dem in Eisenmeteoriten gemessenen ${ }^{4} \mathrm{He} /{ }^{3} \mathrm{He}$-Verhältnis - in erster Linie um durch Sekundärteilchen niedriger Energie produzierte

8 E. Vilcsek U. H. Ẅ̈nke, Z. Naturforschg. 20 a, 1282 [1965].

9 H. Hintenderger, E. Vilcsek u. H. Wänke, Z. Naturforschg. 19 a, 219 [1964].
$\alpha$-Teilchen handelt, deren Energie nicht ausreicht, um eine gleichmäßige Verteilung dieses Heliums in allen Mineralkomponenten zu bewirken (siehe Abschnitt 2 a) .

\section{Diskussion}

$$
\text { a) }{ }^{3} \mathrm{He}
$$

Wie ersichtlich, weichen die ${ }^{3} \mathrm{He}-$ Konzentrationen in den verschiedenen Komponenten eines Meteoriten teilweise recht beträchtlich voneinander ab. Überein-

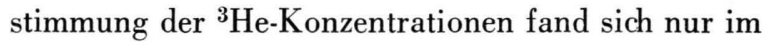
Olivin und Pyroxen der Meteorite $\mathrm{B}$ ath und $\mathrm{O} \mathrm{ch}$ a n sk. Bei diesen beiden Meteoriten kann man auch sicher sein, daß keine Diffusionsverluste von Helium aufgetreten sind, da beide ein hohes U-Th-He-Alter und ein innerhalb der Fehlergrenzen übereinstimmendes K/Ar-Alter besitzen (Tab. 2).

Olivin und Pyroxen sind hinsichtlich der Targetelemente so ähnlich, daß man gleiche ${ }^{3} \mathrm{He}$-Produktion erwarten sollte. Wir können somit schließen, daß in allen Fällen, in denen die ${ }^{3} \mathrm{He}-$ Konzentration im Pyroxen unterhalb der des Olivins liegt, Diffusionsverluste aufgetreten sind. Einzig für den Meteoriten Rose City ergab sich im Pyroxen eine höhere ${ }^{3} \mathrm{He}-K o n z e n t r a t i o n$ als im Olivin. Wir haben hierfür keine Erklärung; da ein Meßfehler nicht ausgeschlossen werden kann, wollen wir diesem Fall zunächst keine allzu hohe Bedeutung zuschreiben. Durch eine Wiederholung der Messung soll noch klargestellt werden, ob es sich um einen reellen Unterschied handelt.

Tab. 2 zeigt die Größe der Diffusionsverluste im Pyroxen. Hierbei gehen wir von der Annahme aus, da $ß$ die im Olivin gemessenen ${ }^{3} \mathrm{He}-K o n z e n t r a t i o n e n$ den wahren Werten entsprechen, d. h. daß in diesem Mineral keine Verluste stattgefunden haben. Diese Annahme ist vor allem durch die Ergebnisse bezüglich des radiogenen Heliums gut bestätigt (siehe 2 b). Wir können nicht entscheiden, ob der Unterschied zwischen Pyroxen und Olivin allein auf unterschiedliche Diffusionskonstanten dieser Minerale oder auch auf eine unterschiedliche Korngrößenverteilung zurückzuführen ist.

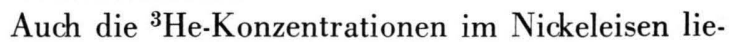
gen tiefer als die entsprechenden Werte für den Olivin. Über das Produktionsverhältnis von ${ }^{3} \mathrm{He}$ im

\footnotetext{
10 D. Heymans, Vortrag C 42, IUPAC-Konferenz, Moskau 1965.
} 
Silikat und Nickeleisen ist nur wenig bekannt. Die ${ }^{3} \mathrm{He}$-Kerne entstehen im Meteoriten als Verdampfungsteilchen, und zwar wird etwa die Hälfte direkt gebildet, der Rest aus Tritium durch nachfolgenden $\beta$-Zerfall. Angaben über die Wirkungsquerschnitte für ${ }^{3} \mathrm{He}$ aus einigen der in Frage kommenden Targetelemente sind nur in der Arbeit von Goebel, Schultes und Z̈̈HRINGER ${ }^{11}$ enthalten, jedoch nur für hohe Energien. Etwas zahlreicher, jedoch voneinander abweichend sind die Messungen der Erzeugungsquerschnitte von Tritium ${ }^{11-13}$. Goebel und Schmidurn ${ }^{14}$ geben für die Tritiumerzeugung durch Protonen von $600 \mathrm{MeV}$ ein Verhältnis von 50 zu 33 für Silikatund Eisenphase an. Dieses Verhältnis entspricht etwa dem Verhältnis der geometrischen Kernquerschnitte und dürfte nicht allzu falsch sein. Wir können diesen Wert auch für das Verhältnis der gesamten ${ }^{3} \mathrm{He}$ Produktion annehmen.

Geiss, Hirt und Oeschger ${ }^{15}$ fanden für den Meteoriten Elenovka in der Metallphase einen Tritiumgehalt von $66 \%$ des Tritiumgehaltes der Silikatphase und haben dabei darauf hingewiesen, daß dies genau dem Verhältnis der vermutlichen Wirkungsquerschnitte entspricht. Diese Annahme ist nicht ganz zutreffend. Currie, Libby und WolfGANG ${ }^{12}$ haben die Anfangsenergien der in Verdampfungsreaktionen entstehenden Tritonen gemessen und für alle Targetelemente von Kohlenstoff bis Blei Energien zwischen 7 und $15 \mathrm{MeV}$ gefunden. Aus der Kernverdampfungstheorie findet man an Eisen eine mittlere Energie der Tritonen von $11 \mathrm{MeV}$, von $13 \mathrm{MeV}$ für ${ }^{3} \mathrm{He}$ und von $14,5 \mathrm{MeV}$ für ${ }^{4} \mathrm{He}^{11}$. Die entsprechenden Reichweiten in Eisen betragen daher $150 \mu$ für Tritonen, $50 \mu$ für ${ }^{3} \mathrm{He}$ und $40 \mu$ für $\alpha$-Teilchen. Normale Chondrite enthalten jedoch nur wenig metallische Nickeleisenkörner mit Durchmessern über $100 \mu$. Falls die Reichweite den Korndurchmesser übersteigt, so ist die Verteilung der bewegten Teilchen zwischen Metall und Silikat eines Chondriten in erster Linie durch das Bremsvermögen dieser beiden Materialien bedingt und nicht durch deren Wirkungsquerschnitte.

Mit einer mittleren chemischen Zusammensetzung des meteoritischen Olivins $(\mathrm{Mg}+\mathrm{Si}=40 \%, \mathrm{Fe}=18 \%$ und $\mathrm{O}_{2}=42 \%$ ) ergibt sich aus dem Bremsvermögen

11 K. Goebel, H.Schultes u. J.Z̈̈hringer, CERN 64-12 [1964]. 12 L. A. Currie, W. F. Libby u. R. I. Wolfgang, Phys. Rev. 101, 1557 [1956].

13 L. A. Currie, Phys. Rev. 114, 878 [1959].

14 K. Goebel u. P. Schmidlin, Radioisotopes in the physical Sciences and Industry, Vol. 1, 13, IAEA, Vienna 1962. für die pro Gewichtseinheit gestoppten Heliummengen im Nickeleisen zu Olivin ein Verhältnis von 0,75 . Dieser Wert gilt praktisch auch für die gesamte Silikatphase. Das Verhältnis der zu erwartenden ${ }^{3} \mathrm{He}-$ Mengen ist also dem Verhältnis der Wirkungsquerschnitte sehr ähnlich. Unabhängig davon, ob die Korngröße kleiner oder größer ist als die Reichweite der Tritonen oder ${ }^{3} \mathrm{He}$-Teilchen, sollten wir also im Nickeleisen eine ${ }^{3} \mathrm{He}-\mathrm{Konzentration}$ von ca. $75 \%$ von der des Olivins erwarten.

Wie aus Tab. 3, Spalte 5 ersichtlich, kommt das

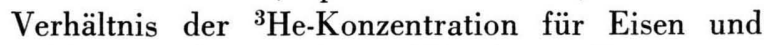
Olivin nur selten an obigen Wert heran. Wir müssen also schließen, daß ${ }^{3} \mathrm{He}$ aus dem chondritischen Eisen verlorengegangen ist. Da jedoch der tiefste gemessene Wert für das Verhältnis ${ }^{3} \mathrm{He}_{\text {Metall }}{ }^{3} \mathrm{He}_{\text {olivin }}$ bei 0,37 liegt (Meteorit Mocs) und damit etwa dem halben theoretischen Wert entspricht, ist der Verdacht naheliegend, daß man es mit einem Verlust von Tritium zu tun hat (ca. $50 \%$ des meteoritischen ${ }^{3} \mathrm{He}$ entstammen dem Zerfall von Tritium). Einen solchen Tritiumverlust haben Hintenberger und WäNKE ${ }^{16}$ schon für den Hexaedriten $\mathrm{Braunau}$ als Ursache für dessen niedriges ${ }^{3} \mathrm{He} /{ }^{4} \mathrm{He}-$ Verhältnis angegeben. Bei den Steinmeteoriten scheint ein solcher Tritiumverlust aus dem Nickeleisen die Regel zu sein. Die oben erwähnte Tritiummessung von Geiss, Hirt und Oeschger ${ }^{15}$ an der Metallphase des Meteoriten Elenovka, die $66 \%$ des Tritiumgehaltes der Silikatphase ergab, steht nur scheinbar im Widerspruch zu diesem Ergebnis. Zunächst gibt es in Tab. 3 auch Meteorite, bei denen der Tritiumverlust klein ist. Geiss, Hirt und Oeschger ${ }^{15}$ geben in ihrer Arbeit keinen Hinweis über die Reinheit der Metallphase. Es ist jedoch sehr schwierig, reine Metallphase zu erhalten; somit würde eine notwendige Korrektur einen niedrigeren Wert ergeben. Fireman und DeFelice ${ }^{17}$ fanden in einer Metallfraktion des Meteoriten B ruderheim nur ca. $30 \%$ des Tritiums der Silikatphase. Diese Autoren schieben aber den Großteil dieser Aktivität auf eine Silikatverunreinigung und kommen zu dem Schluß, $\mathrm{da} \beta$ aus der Metallphase dieses Meteoriten praktisch das gesamte Tritium verlorenging. Unter den Eisenmeteoriten ist noch kein Fall bekannt geworden, der

15 J. Geiss, B. Hirt u. H. Oeschger, Helv. Phys. Acta 33, 590 [1960].

16 H. Hintenberger u. H. Wänke, Z. Naturforschg. 19 a, 210 [1964].

17 E. L. Fireman u. J. DeFelice, J. Geophys. Res. 66, 3547 [1961]. 


\begin{tabular}{|c|c|c|c|c|c|c|c|c|c|c|c|c|c|c|c|}
\hline \multirow{2}{*}{ Meteorit } & \multirow{2}{*}{$\underset{\text { Metall }}{\mathrm{Fe}+\mathrm{Ni}}$} & $\mathrm{FeS}$ & Olivin & $\begin{array}{c}\text { Pyro- } \\
\text { xen }\end{array}$ & \multirow[t]{2}{*}{$\Sigma$} & ${ }^{3} \mathrm{He}$ & ${ }^{4} \mathrm{He}$ & ${ }^{20} \mathrm{Ne}$ & ${ }^{21} \mathrm{Ne}$ & \multirow[t]{2}{*}{${ }^{22} \mathrm{Ne}$} & \multirow[t]{2}{*}{${ }^{3} \mathrm{He}$} & \multirow{2}{*}{\multicolumn{3}{|c|}{ 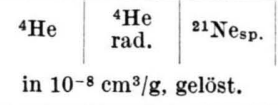 }} & \multirow[t]{2}{*}{$\frac{{ }^{3} \mathrm{He}}{{ }^{21} \mathrm{Ne}_{\mathrm{sp}}}$} \\
\hline & & \multicolumn{3}{|c|}{ Gewichtsprozent } & & \multicolumn{4}{|c|}{ in $10^{-8} \mathrm{~cm}^{3} / \mathrm{g}$ Met. } & & & & & & \\
\hline \multicolumn{16}{|c|}{ Hypersthen-Chondrite } \\
\hline \multirow{3}{*}{$\begin{array}{l}\text { Akaba } \\
\mathrm{CuCl}_{2} \\
\mathrm{Br} \\
\mathrm{HCl} \\
\mathrm{Rü} \\
\Sigma \\
\text { Lit. }{ }^{4}\end{array}$} & $\begin{array}{l}7,91 \\
1.78\end{array}$ & 4,29 & $\begin{array}{c}0,50 \\
1,46 \\
33,1\end{array}$ & 47,1 & $\begin{array}{c}8.41 \\
7,53 \\
33,1 \\
47,1 \\
\end{array}$ & $\begin{array}{l}2,35 \\
2,95 \\
15,8 \\
18,9\end{array}$ & $\begin{array}{c}24.9 \\
70,6 \\
269 \\
421\end{array}$ & $\begin{array}{l}0,14 \\
0,26 \\
2,20 \\
2,28\end{array}$ & $\begin{array}{l}0,06 \\
0,24 \\
2,27 \\
2,54\end{array}$ & $\begin{array}{l}0,08 \\
0,29 \\
2.54 \\
2,88\end{array}$ & \multirow[t]{3}{*}{$\begin{array}{l}28 \\
39 \\
48 \\
40\end{array}$} & \multirow[t]{3}{*}{$\begin{array}{l}300 \\
940 \\
820 \\
890\end{array}$} & \multirow[t]{3}{*}{$\begin{array}{l}190 \\
740 \\
570 \\
790\end{array}$} & \multirow[t]{3}{*}{$\begin{array}{l}0,33^{*} \\
3.2 \\
6,9 \\
5,4\end{array}$} & \multirow[t]{3}{*}{$\begin{array}{l}85 \\
12 \\
7,0 \\
7,4\end{array}$} \\
\hline & 9,69 & 4,29 & 35,1 & 47,1 & 96,1 & 40,0 & 786 & 4,88 & 5,11 & 5,79 & & & & & \\
\hline & & & & & & 40,9 & 805 & 4,94 & 5,42 & 6,14 & & & & & \\
\hline \multicolumn{2}{|c|}{ Bruderheim } & & & & & & & & & & \multirow{4}{*}{$\begin{array}{l}30 \\
33 \\
60 \\
42\end{array}$} & \multirow{4}{*}{$\begin{array}{l}310 \\
450 \\
680 \\
340\end{array}$} & \multirow{4}{*}{$\begin{array}{l}190 \\
280 \\
380 \\
130\end{array}$} & \multirow{4}{*}{$\begin{array}{c}0,80^{*} \\
2,9 \\
12,2 \\
7,2\end{array}$} & \multirow{4}{*}{$\begin{array}{c}38 \\
11 \\
4,9 \\
5,8\end{array}$} \\
\hline $\begin{array}{l}\mathrm{CuCl}_{2} \\
\mathrm{Br} \\
\mathrm{HCl} \\
\mathrm{Ru}\end{array}$ & \begin{tabular}{|l|}
8,75 \\
2,09
\end{tabular} & 5,35 & $\begin{array}{c}0,82 \\
3,79 \\
32,4\end{array}$ & 41,3 & $\begin{array}{l}9,57 \\
11.23 \\
32,4 \\
41,3 \\
\end{array}$ & $\begin{array}{l}2,90 \\
3,68 \\
19,3 \\
17,2 \\
\end{array}$ & $\begin{array}{l}29,3 \\
50,7 \\
220 \\
142\end{array}$ & $\begin{array}{l}0,40 \\
0,39 \\
3,75 \\
2,55\end{array}$ & $\begin{array}{l}0,17 \\
0,33 \\
3,95 \\
2,98\end{array}$ & $\begin{array}{l}0,20 \\
0,40 \\
4,09 \\
3,18\end{array}$ & & & & & \\
\hline \multirow{2}{*}{$\begin{array}{l}\sum \\
\text { Lit. }^{4}\end{array}$} & 10,84 & 5,35 & 37,0 & 41,3 & 94,5 & 43,1 & 442 & 7,09 & 7,43 & 7,87 & & & & & \\
\hline & & & & & & 47,4 & 526 & 7,21 & 8,17 & 8.69 & & & & & \\
\hline \multirow{3}{*}{\begin{tabular}{|l|} 
Goodland \\
$\mathrm{CuCl}_{2}$ \\
$\mathrm{Br}$ \\
$\mathrm{HCl}$ \\
$\mathrm{Rü}$ \\
$\Sigma$ \\
Lit. $^{5}$ \\
\end{tabular}} & $\begin{array}{l}787 \\
1,20\end{array}$ & 5,78 & $\begin{array}{c}0,17 \\
2,33 \\
29,7\end{array}$ & 46,6 & $\begin{array}{l}8,04 \\
9,31 \\
29,7 \\
46,6\end{array}$ & $\begin{array}{l}1,22 \\
1,51 \\
884 \\
9,17\end{array}$ & $\begin{array}{l}13,2 \\
13,2 \\
82,7 \\
97,8 \\
\end{array}$ & $\begin{array}{l}1,18 \\
- \\
1,74 \\
2,34\end{array}$ & $\begin{array}{c}0,033 \\
- \\
1,84 \\
2,55\end{array}$ & $\begin{array}{l}0,14 \\
- \\
1,88 \\
2,64 \\
\end{array}$ & \multirow[t]{3}{*}{$\begin{array}{l}15 \\
16 \\
30 \\
20\end{array}$} & $\begin{array}{l}160 \\
140 \\
280 \\
210\end{array}$ & $\begin{array}{r}100 \\
60 \\
130 \\
110\end{array}$ & $\begin{array}{l}0,27 * \\
-\overline{2} \\
5,5\end{array}$ & $\begin{array}{l}56 \\
- \\
4,8 \\
3,6\end{array}$ \\
\hline & 9,07 & 5,78 & 32,2 & 46,6 & 93,7 & 20,7 & 207 & 5,28 & 4,44 & 4,68 & & & & & \\
\hline & & & & & & 24,2 & 220 & 6,36 & 5,37 & 5,79 & & & & & \\
\hline Hayes $\mathrm{Ce}$ & enter & & & & & & & & & & & & & & \\
\hline $\begin{array}{l}\mathrm{CuCl}_{2} \\
\mathrm{Br} \\
\mathrm{HCl} \\
\mathrm{Rü}\end{array}$ & $\begin{array}{l}8,65 \\
1,44\end{array}$ & 5,25 & $\begin{array}{c}0,24 \\
3,31 \\
26,5\end{array}$ & 51,5 & $\begin{array}{c}8,89 \\
10,0 \\
26,5 \\
51,5\end{array}$ & $\begin{array}{c}2,70 \\
1,53 \\
13,7 \\
22,8 \\
\end{array}$ & $\begin{array}{r}14,6 \\
25,8 \\
94,8 \\
171\end{array}$ & $\begin{array}{l}0,08 \\
0,38 \\
3,38 \\
5,47\end{array}$ & $\begin{array}{l}0,09 \\
0,42 \\
3,70 \\
5,51\end{array}$ & $\begin{array}{l}0,09 \\
0,44 \\
3,80 \\
5,81\end{array}$ & $\begin{array}{l}30 \\
15 \\
52 \\
44\end{array}$ & $\begin{array}{l}160 \\
260 \\
360 \\
330\end{array}$ & $\begin{array}{c}40 \\
180 \\
100 \\
110\end{array}$ & $\begin{array}{c}0,7^{*} \\
4,2 \\
14,0 \\
10,7\end{array}$ & $\begin{array}{l}43 \\
36 \\
37 \\
4,1\end{array}$ \\
\hline$\Sigma$ & 10,09 & 5,25 & 30,0 & 51,5 & 96,9 & 40,7 & 305 & 9,31 & 9,72 & 10,14 & & & & & \\
\hline $\mathrm{Lit}^{4}$ & & & & & & 49,0 & 348 & 14,0 & 10,0 & 10,0 & & & & & \\
\hline $\begin{array}{l}\text { Holbrook } \\
\mathrm{CuCl}_{2} \\
\mathrm{Br} \\
\mathrm{HCl} \\
\mathrm{Rü}\end{array}$ & \begin{tabular}{|l|} 
\\
5,74 \\
1,55
\end{tabular} & 5,43 & $\begin{array}{c}0,25 . \\
2,37 \\
36,7\end{array}$ & 45,8 & $\begin{array}{c}5.99 \\
9,35 \\
36,7 \\
45,8 \\
\end{array}$ & $\begin{array}{l}1,26 \\
9,12 \\
9,59 \\
\end{array}$ & $\begin{array}{r}30 \\
173 \\
468 \\
845 \\
\end{array}$ & $\begin{array}{l}0,26 \\
0,48 \\
2,29 \\
2,90\end{array}$ & $\begin{array}{l}0,034 \\
0,19 \\
2,41 \\
2,73\end{array}$ & $\begin{array}{l}0,06 \\
0,24 \\
2,43 \\
2,94\end{array}$ & $\begin{array}{l}14^{-} \\
25 \\
21\end{array}$ & $\begin{array}{r}500 \\
1860 \\
1270 \\
1850\end{array}$ & $\begin{array}{c}-\overline{790} \\
1140 \\
1740\end{array}$ & $\begin{array}{l}0,32^{*} \\
2,0 \\
6,6 \\
6,0\end{array}$ & $\begin{array}{l}\overline{7,0} \\
3,8 \\
3,5\end{array}$ \\
\hline$\Sigma$ & 7,29 & 5,43 & 39,3 & 45,8 & 97,8 & 20,3 & 1517 & 5,93 & 5,36 & 5,67 & & & & & \\
\hline Lit. ${ }^{4}$ & & & & & & 24,1 & 1740 & 5.42 & 5,74 & 6,31 & & & & & \\
\hline $\begin{array}{l}\text { Kiel } \\
\mathrm{CuCl}_{2} \\
\mathrm{Br} \\
\mathrm{HCl} \\
\mathrm{Ru}\end{array}$ & $\begin{array}{l}7,86 \\
1,47\end{array}$ & 4,86 & $\begin{array}{c}0,17 \\
1,25 \\
23,6\end{array}$ & 56,0 & $\begin{array}{c}8,03 \\
7,58 \\
23,6 \\
56,0 \\
\end{array}$ & $\begin{array}{r}1,54 \\
1,26 \\
6,55 \\
11,8 \\
\end{array}$ & $\begin{array}{c}45,1 \\
185 \\
238 \\
590\end{array}$ & $\begin{array}{l}0,65 \\
0,50 \\
0,88 \\
1,38\end{array}$ & $\begin{array}{l}0,046 \\
0,12 \\
0,81 \\
1,44\end{array}$ & $\begin{array}{l}0,10 \\
0,17 \\
0,98 \\
1,72\end{array}$ & $\begin{array}{l}19 \\
17 \\
28 \\
21\end{array}$ & $\begin{array}{r}560 \\
2430 \\
1010 \\
1050\end{array}$ & $\begin{array}{r}480 \\
2340 \\
870 \\
940\end{array}$ & $\begin{array}{l}0,51^{*} \\
16 \\
3,4 \\
2,6\end{array}$ & $\begin{array}{l}37 \\
11 \\
8,2 \\
8,1\end{array}$ \\
\hline$\Sigma$ & 9,33 & 4,86 & 25,0 & 56,0 & 95,2 & 21,2 & 1058 & 3,41 & 2,45 & 2,97 & & & & & \\
\hline Lit. ${ }^{5}$ & & & & & & 26,5 & 1256 & 3,08 & 2,50 & 3,26 & & & & & \\
\hline $\begin{array}{l}\text { Mocs } \\
\mathrm{CuCl}_{2} \\
\mathrm{Br} \\
\mathrm{HCl} \\
\mathrm{Rül}\end{array}$ & $\begin{array}{c}6,92 \\
0,91\end{array}$ & 5,31 & $\begin{array}{l}0,44 \\
0,95 \\
31,9\end{array}$ & 49,3 & $\begin{array}{c}7,36 \\
7,17 \\
319 \\
49,3\end{array}$ & $\begin{array}{c}0,78 \\
0,22 \\
9,37 \\
9,92\end{array}$ & $\begin{array}{c}22,8 \\
9,9 \\
232 \\
313\end{array}$ & $\begin{array}{r}0,34 \\
0,056 \\
2,19 \\
2,51\end{array}$ & $\begin{array}{c}0,048 \\
0,037 \\
2,10 \\
2,63\end{array}$ & $\begin{array}{c}0,071 \\
0,037 \\
2,23 \\
2,87 \\
\end{array}$ & $\begin{array}{c}11 \\
3,1 \\
30 \\
20\end{array}$ & $\begin{array}{c}310 \\
140 \\
730 \\
640\end{array}$ & $\begin{array}{c}270 \\
120 \\
580 \\
540\end{array}$ & $\begin{array}{c}0,28^{*} \\
0,47 \\
6,6 \\
5,3\end{array}$ & $\begin{array}{l}39 \\
6,4 \\
4,6 \\
3,8\end{array}$ \\
\hline$\Sigma$ & 7,83 & 5,31 & 33,3 & 49,3 & 95,7 & 20,3 & 577 & 5,10 & 4,82 & 5,14 & & & & & \\
\hline Lit. ${ }^{4}$ & & & & & & 23,3 & 750 & 5,41 & 5,41 & 5,64 & & & & & \\
\hline New $C$ & icora & & & & & & & & & & & & & & \\
\hline $\begin{array}{l}\mathrm{CuCl}_{2} \\
\mathrm{Br} \\
\mathrm{HCl} \\
\mathrm{Rü}\end{array}$ & $\begin{array}{l}8,74 \\
0,52\end{array}$ & 6,16 & $\begin{array}{c}0,37 \\
5,4 \\
29,0\end{array}$ & 45,9 & $\begin{array}{c}9,11 \\
153 \\
29.0 \\
45.9\end{array}$ & $\begin{array}{l}0,24 \\
0,47 \\
1,37 \\
1,44\end{array}$ & $\begin{array}{c}20,6 \\
22,4 \\
119 \\
57,2\end{array}$ & $\begin{array}{l}0,56 \\
4,89 \\
0,41 \\
0,75\end{array}$ & $\begin{array}{l}0,015 \\
0,20 \\
0,40 \\
0,47\end{array}$ & $\begin{array}{l}0,07 \\
0,62 \\
0,40 \\
0,50\end{array}$ & $\begin{array}{l}2,6 \\
3,1 \\
4,7 \\
3,1\end{array}$ & $\begin{array}{l}250 \\
150 \\
410 \\
120\end{array}$ & $\begin{array}{l}240 \\
130 \\
390 \\
100\end{array}$ & $\begin{array}{l}0,09 * \\
1,2 \\
1,38 \\
1,03\end{array}$ & $\begin{array}{c}30 \\
2,6 \\
3,4 \\
3,0\end{array}$ \\
\hline$\Sigma$ & 9,26 & 6,16 & 38,0 & 45,9 & 99,3 & 3,52 & 219 & 6,61 & 1,06 & 1,59 & & & & & \\
\hline Lit. ${ }^{5}$ & & & & & & 4,33 & 255 & 1,48 & 1,09 & 1,01 & & & & & \\
\hline
\end{tabular}

Tab. 1 . 


\begin{tabular}{|c|c|c|c|c|c|c|c|c|c|c|c|c|c|c|c|}
\hline \multirow[t]{2}{*}{ Meteorit } & \multirow{2}{*}{$\begin{array}{l}\mathrm{Fe}+\mathrm{Ni} \\
\text { Metall }\end{array}$} & \multirow[t]{2}{*}{ FeS } & Olivin & $\begin{array}{c}\text { Pyro- } \\
\text { xen }\end{array}$ & $\Sigma$ & ${ }^{3} \mathrm{He}$ & ${ }^{4} \mathrm{He}$ & ${ }^{20} \mathrm{Ne}$ & ${ }^{21} \mathrm{Ne}$ & ${ }^{22} \mathrm{Ne}$ & \multirow[t]{2}{*}{${ }^{3} \mathrm{He}$} & & $\begin{array}{l}{ }^{4} \mathrm{He} \\
\text { rad. }\end{array}$ & ${ }^{21} \mathrm{Ne}$ sp. & $\frac{{ }^{3} \mathrm{He}}{{ }^{21} \mathrm{Ne}_{\mathrm{sp}} .}$ \\
\hline & & & \multicolumn{3}{|c|}{ Gewichtsprozent } & \multicolumn{5}{|c|}{ in ${ }^{-8} \mathrm{~cm}^{3} / \mathrm{g}$ Met. } & & \multicolumn{4}{|c|}{ in $10^{-8} \mathrm{~cm}^{3} / \mathrm{g}$ gelöst } \\
\hline \multicolumn{16}{|c|}{ Hypersthen - Chondrite } \\
\hline \multirow{3}{*}{ 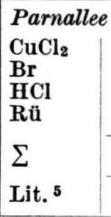 } & $\begin{array}{l}6,28 \\
0,41\end{array}$ & 5,15 & $\begin{array}{c}0,43 \\
5,0 \\
26,0\end{array}$ & 44,3 & $\begin{array}{c}6,71 \\
10,6 \\
26,0 \\
44,3\end{array}$ & $\begin{array}{l}0,17 \\
0,22 \\
1,28 \\
1,89\end{array}$ & $\begin{array}{l}14,4 \\
20,7 \\
180 \\
194\end{array}$ & $\begin{array}{l}0,62 \\
0,23 \\
0,67 \\
1,14\end{array}$ & $\begin{array}{l}0,011 \\
0,14 \\
0,63 \\
0,87\end{array}$ & $\begin{array}{l}0,07 \\
0,16 \\
0,67 \\
0,95\end{array}$ & \multirow[t]{3}{*}{$\begin{array}{l}3,6 \\
2,0 \\
4,9 \\
4,3\end{array}$} & \multirow[t]{3}{*}{$\begin{array}{l}300 \\
190 \\
690 \\
440\end{array}$} & \multirow[t]{3}{*}{$\begin{array}{l}290 \\
180 \\
670 \\
420\end{array}$} & \multirow[t]{3}{*}{$\begin{array}{l}0,09^{*} \\
1,3 \\
2,4 \\
2,0\end{array}$} & \multirow[t]{3}{*}{$\begin{array}{c}40 \\
1,5 \\
2,0 \\
2,2\end{array}$} \\
\hline & 6,69 & 5,15 & 31,4 & 44,3 & 87,6 & 3,56 & 409 & 2,66 & 1,65 & 1,85 & & & & & \\
\hline & & & & & & 6,37 & 697 & 2,59 & 2,33 & 2,52 & & & & & \\
\hline \multirow{3}{*}{\begin{tabular}{|l|} 
Walters \\
$\mathrm{CuCl}_{2}$ \\
$\mathrm{Br}_{2}$ \\
$\mathrm{HCl}$ \\
$\mathrm{Rü}$ \\
$\Sigma$ \\
Lit. $^{5}$ \\
\end{tabular}} & $\begin{array}{l}8,10 \\
0,39\end{array}$ & 6,09 & $\begin{array}{c}0,33 \\
0,66 \\
32,9\end{array}$ & 49,7 & $\begin{array}{c}8,43 \\
7,14 \\
32,9 \\
49,7\end{array}$ & $\begin{array}{l}0,19 \\
0.20 \\
1,71 \\
1,79\end{array}$ & $\begin{array}{r}13,3 \\
14,1 \\
114,5 \\
57,3 \\
\end{array}$ & $\begin{array}{l}0,97 \\
2,6 \\
0,84 \\
0,80\end{array}$ & $\begin{array}{l}0,023 \\
0,023 \\
0,73 \\
0,69 \\
\end{array}$ & $\begin{array}{l}0,11 \\
0,26 \\
0,75 \\
0,72 \\
\end{array}$ & \multirow[t]{3}{*}{$\begin{array}{l}2,7 \\
2,7 \\
5,2 \\
3,6\end{array}$} & \multirow[t]{3}{*}{$\begin{array}{l}190 \\
190 \\
350 \\
120\end{array}$} & \multirow[t]{3}{*}{$\begin{array}{l}180 \\
180 \\
320 \\
100\end{array}$} & \multirow[t]{3}{*}{$\begin{array}{l}0,17^{*} \\
0,27 \\
2,2 \\
1,4\end{array}$} & \multirow[t]{3}{*}{$\begin{array}{c}16 \\
10 \\
2,4 \\
2,6\end{array}$} \\
\hline & 8,49 & 6,09 & 33,9 & 49,7 & 98,2 & 3,89 & 199 & 5,21 & $1: 47$ & 1,84 & & & & & \\
\hline & & & & & & 4,85 & 224 & 1,79 & 1,28 & 1,38 & & & & & \\
\hline \multicolumn{16}{|c|}{ Bronzit - Chondrite } \\
\hline \multirow{3}{*}{$\begin{array}{l}\text { Bath } \\
\mathrm{CuCl}_{2} \\
\mathrm{Br} \\
\mathrm{HCl} \\
\mathrm{Rü} \\
\Sigma \\
\text { Lit. }{ }^{\circ} \\
\end{array}$} & $\begin{array}{c}18,3 \\
0,73\end{array}$ & 4,98 & $\begin{array}{c}0,30 \\
1,91 \\
23,3\end{array}$ & 48,2 & $\begin{array}{c}18,6 \\
7,62 \\
23,3 \\
48,2 \\
\end{array}$ & $\begin{array}{l}1,08 \\
0,67 \\
3,08 \\
6,22 \\
\end{array}$ & $\begin{array}{c}39,8 \\
61,3 \\
213 \\
878 \\
\end{array}$ & $\begin{array}{l}0,21 \\
0,15 \\
1,24 \\
0,97\end{array}$ & $\begin{array}{l}0,073 \\
0,10 \\
0,81 \\
1,16\end{array}$ & $\begin{array}{l}0,09 \\
0,13 \\
0,89 \\
1,26\end{array}$ & \multirow[t]{3}{*}{$\begin{array}{r}5,8 \\
8,8 \\
13,2 \\
12,9\end{array}$} & \multirow[t]{3}{*}{$\begin{array}{r}210 \\
810 \\
910 \\
1820\end{array}$} & \multirow[t]{3}{*}{$\begin{array}{r}190 \\
770 \\
840 \\
1760\end{array}$} & \multirow[t]{3}{*}{$\begin{array}{l}0,34^{*} \\
1,32 \\
3,5 \\
2,4\end{array}$} & $\begin{array}{c}17 \\
6,7 \\
3,8 \\
5,4\end{array}$ \\
\hline & 19,0 & 4,98 & 25,5 & 48,2 & 97,7 & 11,1 & 1192 & 2,57 & 2,14 & 2,37 & & & & & \\
\hline & & & & & & 10,2 & 1258 & 2,39 & 1,93 & 2,11 & & & & & \\
\hline Beddgeler & & & & & & & & & & & & & & & \\
\hline $\begin{array}{l}\mathrm{CuCl}_{2} \\
\mathrm{Br} \\
\mathrm{HCl} \\
\mathrm{Ru}\end{array}$ & $\begin{array}{c}18,4 \\
0,84\end{array}$ & 5,39 & $\begin{array}{c}0,31 \\
9,06 \\
19,9\end{array}$ & 44,9 & $\begin{array}{l}18,7 \\
15,3 \\
19,9 \\
44,9\end{array}$ & $\begin{array}{l}1,01 \\
0,68 \\
2,08 \\
2,88 \\
\end{array}$ & $\begin{array}{r}27,8 \\
23,7 \\
108 \\
48,7 \\
\end{array}$ & $\begin{array}{l}0,20 \\
0,19 \\
0,46 \\
0,70 \\
\end{array}$ & $\begin{array}{l}0,035 \\
0,18 \\
0,49 \\
0,72\end{array}$ & $\begin{array}{l}0,05 \\
0,20 \\
0,52 \\
0,78\end{array}$ & $\begin{array}{r}5,4 \\
4,5 \\
10,4 \\
6,4\end{array}$ & $\begin{array}{l}150 \\
160 \\
540 \\
110\end{array}$ & $\begin{array}{r}130 \\
140 \\
490 \\
80\end{array}$ & $\begin{array}{l}0,15 * \\
1,18 \\
2,5 \\
1,6\end{array}$ & $\begin{array}{l}36 \\
3,8 \\
4,2 \\
4,0\end{array}$ \\
\hline$\Sigma$ & 19,2 & 5,39 & 29,3 & 44,9 & 98,8 & 6,65 & 208 & 1,55 & 1,43 & 1,55 & & & & & \\
\hline Lit. ${ }^{4}$ & & & & & & 7,44 & 243 & 1,67 & 1,66 & 1,81 & & & & & \\
\hline Breitsche & id (hell) & & & & & & & & & & & & & & \\
\hline $\begin{array}{l}\mathrm{FeCl}_{3} \\
\mathrm{Br} \\
\mathrm{HCl} \\
\mathrm{Rü}\end{array}$ & 17,1 & 5,6 & $\begin{array}{c}0,48 \\
3,30 \\
24,6\end{array}$ & 49,3 & $\begin{array}{r}17,6 \\
8,9 \\
24,6 \\
49,3\end{array}$ & $\begin{array}{c}8,8 \\
-\overline{-} \\
16,5 \\
7,16\end{array}$ & $\begin{array}{l}91,0 \\
- \\
291 \\
267\end{array}$ & $\begin{array}{l}0,374 \\
-\overline{3}, 75 \\
4,30\end{array}$ & $\begin{array}{l}0,196 \\
-\overline{3,98} \\
\mathbf{3 , 6 4}\end{array}$ & $\begin{array}{l}0,214 \\
-\overline{4,25} \\
4,04\end{array}$ & $\begin{array}{l}50 \\
-7 \\
14,5\end{array}$ & $\begin{array}{r}520 \\
1180 \\
540\end{array}$ & $\begin{array}{l}320 \\
\overline{840} \\
470\end{array}$ & $\begin{array}{r}1,11 \\
- \\
16,2 \\
7,4\end{array}$ & $\begin{array}{l}\frac{45}{-} \\
4,1 \\
1,9\end{array}$ \\
\hline$\Sigma$ & 17,1 & 5,6 & 28,4 & 49,3 & 100,4 & 32,5 & 649 & 8,42 & 7,82 & 8,50 & & & & & \\
\hline Lit. ${ }^{4}$ & & & & & & 35,3 & 608 & 7,95 & 9,25 & 9,94 & & & & & \\
\hline \begin{tabular}{|l|} 
Cullison \\
$\mathrm{CuCl}_{2}$ \\
$\mathrm{Br}$ \\
$\mathbf{H C l}$ \\
$\mathbf{R u}$
\end{tabular} & $\begin{array}{c}16,7 \\
0,24\end{array}$ & 4,14 & $\begin{array}{c}0,35 \\
0,79 \\
20,7\end{array}$ & 51,6 & $\begin{array}{c}17,1 \\
5,17 \\
20,7 \\
51,6 \\
\end{array}$ & $\begin{array}{l}0,13 \\
0,005 \\
0,39 \\
0,30 \\
\end{array}$ & $\begin{array}{r}28,7 \\
1,1 \\
168 \\
91,1 \\
\end{array}$ & $\begin{array}{l}2,25 \\
0,158 \\
0,58 \\
1,17 \\
\end{array}$ & $\begin{array}{l}0,013 \\
0,002 \\
0,21 \\
0,43 \\
\end{array}$ & $\begin{array}{l}0,22 \\
0,02 \\
0,25 \\
0,50 \\
\end{array}$ & $\begin{array}{l}0,76 \\
0,07 \\
1,88 \\
0,58\end{array}$ & $\begin{array}{r}168 \\
16 \\
810 \\
177\end{array}$ & $\begin{array}{r}165 \\
16 \\
800 \\
174\end{array}$ & $\begin{array}{l}0,03 \\
0,03 \\
1,01 \\
0,83\end{array}$ & $\begin{array}{l}25 \\
2 \\
1,9 \\
0,7\end{array}$ \\
\hline$\Sigma$ & 16,9 & 4,14 & 21,8 & 51,6 & 94,6 & 0,83 & 289 & 4,16 & 0,66 & 0,99 & & & & & \\
\hline Lit. ${ }^{5}$ & & & & & & 0,79 & 293 & 1,42 & 0,55 & 0,65 & & & & & \\
\hline \begin{tabular}{|l|} 
Ochansk \\
$\mathrm{CuCl}_{\mathbf{2}}$ \\
$\mathrm{Br}$ \\
$\mathbf{H C l}$ \\
$\mathbf{R u}$
\end{tabular} & $\begin{array}{c}19,8 \\
1,03\end{array}$ & 4,81 & $\begin{array}{c}0,30 \\
2,14 \\
18,3\end{array}$ & 52,4 & $\begin{array}{c}20,1 \\
7,98 \\
18,3 \\
52,4\end{array}$ & $\begin{array}{l}1,67 \\
0,74 \\
2,22 \\
6,43\end{array}$ & $\begin{array}{c}51,4 \\
117 \\
194 \\
1120\end{array}$ & $\begin{array}{l}0,67 \\
0,07 \\
0,68 \\
1,94\end{array}$ & $\begin{array}{l}0,040 \\
0,06 \\
0,58 \\
1,62\end{array}$ & $\begin{array}{l}0,11 \\
0,08 \\
0,61 \\
1,75\end{array}$ & $\begin{array}{r}8,3 \\
9,3 \\
12,2 \\
12,3\end{array}$ & $\begin{array}{r}260 \\
1480 \\
1060 \\
1940\end{array}$ & $\begin{array}{r}230 \\
1430 \\
1000 \\
1880\end{array}$ & $\begin{array}{l}0,14^{*} \\
0,76 \\
3,2 \\
3,1\end{array}$ & $\begin{array}{c}59 \\
12 \\
3,8 \\
4,0\end{array}$ \\
\hline$\Sigma$ & 20,8 & 4,81 & 20,8 & 52,4 & 98,8 & 11,8 & 1380 & 3,36 & 2,30 & 2,55 & & & & & \\
\hline Lit. $^{5}$ & & & & & & 13,2 & 1856 & - & 2,34 & - & & & & & \\
\hline Pantar & hell) & & & & & & & & & & & & & & \\
\hline $\begin{array}{l}\mathrm{CuCl}_{2} \\
\mathrm{Br} \\
\mathbf{H C l} \\
\mathrm{Rü}\end{array}$ & $\begin{array}{c}17,1 \\
0,65\end{array}$ & 5,02 & $\begin{array}{c}0,22 \\
5,93 \\
21,4\end{array}$ & 48,4 & $\begin{array}{l}17,3 \\
11,6 \\
21,4 \\
48,4 \\
\end{array}$ & $\begin{array}{l}0,40 \\
0,17 \\
1,19 \\
1,76 \\
\end{array}$ & $\begin{array}{c}33,0 \\
44,5 \\
225 \\
700\end{array}$ & $\begin{array}{l}0,31 \\
0,06 \\
0,35 \\
0,59\end{array}$ & $\begin{array}{l}0,026 \\
0,06 \\
0,37 \\
0,61 \\
\end{array}$ & $\begin{array}{l}0,05 \\
0,08 \\
0,37 \\
0,64\end{array}$ & $\begin{array}{l}2,84 \\
1,43 \\
5,6 \\
3,6\end{array}$ & $\begin{array}{r}190 \\
380 \\
1050 \\
1450\end{array}$ & $\begin{array}{r}180 \\
370 \\
1020 \\
1430\end{array}$ & $\begin{array}{l}0,12 \\
0,68 \\
1,73 \\
1,26\end{array}$ & $\begin{array}{c}24 \\
2,1 \\
3,2 \\
2,9\end{array}$ \\
\hline$\Sigma$ & 17,8 & 5,02 & 27,6 & 48,4 & 98,7 & 3,52 & 1000 & 1,31 & 1,07 & 1,14 & & & & & \\
\hline Lit. ${ }^{5}$ & & & & & & 4,1 & 1036 & 0,74 & 1,05 & 1,04 & & & & & \\
\hline
\end{tabular}

Tab. 1. 


\begin{tabular}{|c|c|c|c|c|c|c|c|c|c|c|c|c|c|c|c|}
\hline \multirow[t]{2}{*}{ Meteorit } & \multirow{2}{*}{$\mid \begin{array}{c}\mathrm{Fe}+\mathrm{Ni} \\
\text { Metall }\end{array}$} & \multirow[t]{2}{*}{ Fes } & Olivin & $\begin{array}{c}\text { Pyro- } \\
\text { xen }\end{array}$ & $\Sigma$ & ${ }^{3} \mathrm{He}$ & ${ }^{4} \mathrm{He}$ & ${ }^{20} \mathrm{Ne}$ & ${ }^{21} \mathrm{Ne}$ & ${ }^{22} \mathrm{Ne}$ & ${ }^{3} \mathrm{He}$ & ${ }^{4} \mathrm{He}$ & $\begin{array}{l}{ }^{4} \mathrm{He} \\
\text { rad. }\end{array}$ & ${ }^{21} \mathrm{Ne}_{\mathrm{sp}}$ & $\frac{{ }^{3} \mathrm{He}}{{ }^{21} \mathrm{Ne}_{\mathrm{sp} .}}$ \\
\hline & & & \multicolumn{3}{|c|}{ Gewichtsprozent } & \multicolumn{5}{|c|}{ in $10^{-8} \mathrm{~cm}^{3} / \mathrm{g}$ Met. } & \multicolumn{5}{|c|}{ in $10^{-8} \mathrm{~cm}^{3} / \mathrm{g}$ gelöst } \\
\hline \multicolumn{16}{|c|}{ Bronzit-Chondrite } \\
\hline \multicolumn{2}{|c|}{ Pultusk (hell) } & & & & & & & & & & \multirow{4}{*}{$\begin{array}{r}7,5 \\
5,9 \\
10,8 \\
9,6\end{array}$} & \multirow{4}{*}{$\begin{array}{r}340 \\
2260 \\
980 \\
1730\end{array}$} & \multirow{4}{*}{$\begin{array}{r}310 \\
2230 \\
930 \\
1680\end{array}$} & \multirow{4}{*}{$\begin{array}{l}0,43^{*} \\
0,63 \\
2,7 \\
2,4\end{array}$} & \multirow{4}{*}{$\begin{array}{c}17 \\
9,4 \\
4,0 \\
4,0\end{array}$} \\
\hline $\begin{array}{l}\mathrm{CuCl}_{2} \\
\mathrm{Br} \\
\mathrm{HCl} \\
\mathrm{Rü}\end{array}$ & 17,0 & 4,38 & $\begin{array}{c}0,49 \\
0,80 \\
22,9\end{array}$ & 49,3 & $\begin{array}{c}17,5 \\
5,18 \\
22,9 \\
49,3\end{array}$ & $\begin{array}{l}1,31 \\
0,47 \\
2,47 \\
4,74\end{array}$ & $\begin{array}{l}60,1 \\
181 \\
227 \\
851\end{array}$ & $\begin{array}{l}0,36 \\
1,71 \\
0,66 \\
1,58\end{array}$ & $\begin{array}{l}0,087 \\
0,047 \\
0,62 \\
1,16\end{array}$ & $\begin{array}{l}0,11 \\
0,21 \\
0,65 \\
1,25\end{array}$ & & & & & \\
\hline$\Sigma$ & 17,0 & 4,38 & 24,2 & 49,3 & 94,9 & 8,99 & 1319 & 4,31 & 1,91 & 2,22 & & & & & \\
\hline Lit. ${ }^{5}$ & & & & & & 6,8 & 1383 & 1,67 & 1,61 & 1,68 & & & & & \\
\hline \multicolumn{2}{|l|}{ Rose City } & & & & & & & & & & \multirow{4}{*}{$\begin{array}{l}33,8 \\
31,2 \\
43,1 \\
57,4\end{array}$} & \multirow{4}{*}{$\begin{array}{l}195 \\
300 \\
300 \\
390\end{array}$} & \multirow{4}{*}{$\begin{array}{r}55 \\
140 \\
80 \\
100\end{array}$} & \multirow{4}{*}{$\begin{array}{c}0,49^{*} \\
4,7 \\
14,3 \\
11,3\end{array}$} & \multirow{4}{*}{$\begin{array}{c}69 \\
6,6 \\
3,0 \\
5,1\end{array}$} \\
\hline $\begin{array}{l}\mathrm{CuCl}_{2} \\
\mathrm{Br} \\
\mathrm{HCl} \\
\mathrm{Rü}\end{array}$ & $\begin{array}{c}18,4 \\
0,52\end{array}$ & 4,77 & $\begin{array}{r}0,20 \\
2,56 \\
17,8\end{array}$ & 51,9 & $\begin{array}{c}18,6 \\
7,85 \\
17,8 \\
51,9\end{array}$ & $\begin{array}{r}6,29 \\
2,45 \\
7,67 \\
29,8 \\
\end{array}$ & $\begin{array}{c}36,3 \\
23,3 \\
52,6 \\
203\end{array}$ & $\begin{array}{l}9,40 \\
3,69 \\
2,97 \\
5,65\end{array}$ & $\begin{array}{l}0,148 \\
0,37 \\
2,55 \\
5,86\end{array}$ & $\begin{array}{l}1,00 \\
0,68 \\
2,46 \\
6,06 \\
\end{array}$ & & & & & \\
\hline$\Sigma$ & 18,9 & 4,77 & 20,6 & 51,9 & 96,2 & 46,2 & 315 & 21,7 & 8,93 & 10,2 & & & & & \\
\hline Lit. ${ }^{6}$ & & & & & & 48,3 & 330 & 9,69 & 9,42 & 9,91 & & & & & \\
\hline \multicolumn{2}{|c|}{ Tabor (hell) } & & & & & & & & & & \multirow{4}{*}{$\begin{array}{r}6,5 \\
6,6 \\
12,0 \\
9,3\end{array}$} & \multirow{4}{*}{$\begin{array}{r}210 \\
850 \\
1170 \\
1830\end{array}$} & \multirow{4}{*}{$\begin{array}{r}180 \\
820 \\
1110 \\
1780\end{array}$} & \multirow{4}{*}{$\begin{array}{l}0,12^{*} \\
0,82 \\
3,5 \\
2,6\end{array}$} & \multirow{4}{*}{$\begin{array}{c}54 \\
8,0 \\
3,4 \\
3,6\end{array}$} \\
\hline $\begin{array}{l}\mathrm{CuCl}_{2} \\
\mathrm{Br} \\
\mathrm{HCl} \\
\mathrm{Rü}\end{array}$ & $\begin{array}{r}16,9 \\
1,99\end{array}$ & 5,35 & $\begin{array}{c}0,41 \\
2,38 \\
23,0\end{array}$ & 50,2 & $\begin{array}{c}17,3 \\
9,72 \\
23,0 \\
50,2 \\
\end{array}$ & $\begin{array}{l}1,12 \\
0,64 \\
2,76 \\
4,65\end{array}$ & $\begin{array}{r}36,2 \\
82,7 \\
270 \\
920 \\
\end{array}$ & $\begin{array}{l}0,54 \\
1,00 \\
1,05 \\
1,41\end{array}$ & $\begin{array}{l}0,036 \\
0,079 \\
0,80 \\
1,29\end{array}$ & $\begin{array}{l}0,08 \\
0,16 \\
0,85 \\
1,35\end{array}$ & & & & & \\
\hline$\Sigma$ & 18,9 & 5,35 & 25,8 & 50,2 & 100,2 & 9,17 & 1309 & 4,00 & 2,21 & 2,44 & & & & & \\
\hline Lit. ${ }^{5}$ & & & & & & 10,9 & 1281 & 0,85 & 1,52 & 1,60 & & & & & \\
\hline $\begin{array}{l}\text { Texline } \\
\mathrm{CuCl}_{2} \\
\mathrm{Br} \\
\mathrm{HCl} \\
\mathrm{Rü}\end{array}$ & $\begin{array}{c}16,0 \\
0,61\end{array}$ & 5,29 & $\begin{array}{c}0,27 \\
7,92 \\
22,4\end{array}$ & 45,1 & $\begin{array}{l}16,3 \\
13,8 \\
22,4 \\
45,1 \\
\end{array}$ & $\begin{array}{l}0,73 \\
1,04 \\
2,54 \\
2,35\end{array}$ & \begin{tabular}{l|}
20,8 \\
36,4 \\
175 \\
139 \\
\end{tabular} & $\begin{array}{l}0,56 \\
0,28 \\
0,70 \\
2,15\end{array}$ & $\begin{array}{l}0,025 \\
0,23 \\
0,68 \\
1,50 \\
\end{array}$ & $\begin{array}{l}0,08 \\
0,25 \\
0,71 \\
1,62 \\
\end{array}$ & \multirow[t]{3}{*}{$\begin{array}{r}4,5 \\
7,5 \\
11,3 \\
5,2\end{array}$} & \multirow[t]{3}{*}{$\begin{array}{l}130 \\
260 \\
780 \\
310\end{array}$} & \multirow[t]{3}{*}{$\begin{array}{l}110 \\
220 \\
720 \\
280\end{array}$} & \multirow[t]{3}{*}{$\begin{array}{l}0,14 \\
1,66 \\
3,1 \\
3,3\end{array}$} & $\begin{array}{c}32 \\
4,5 \\
3,7 \\
1,6\end{array}$ \\
\hline$\Sigma$ & 16,6 & 5,29 & 30,6 & 45,1 & 97,6 & 6,90 & 370 & 3,69 & 2,44 & 2,66 & & & & & \\
\hline Lit. ${ }^{5}$ & & & & & & 9,85 & 388 & 3,19 & 2,15 & 2,36 & & & & & \\
\hline Amphote & ritischer & hondri & & & & & & & & & & & & & \\
\hline Dhurmsal & & & & & & & & & & & & & & & \\
\hline $\begin{array}{l}\mathrm{CuCl}_{2} \\
\mathrm{Br} \\
\mathrm{HCl} \\
\mathrm{Rü}\end{array}$ & $\begin{array}{l}3,24 \\
0,74\end{array}$ & 4,63 & $\begin{array}{l}0,49 \\
1,69 \\
35,5\end{array}$ & 45,0 & $\begin{array}{c}3,73 \\
7,06 \\
35,5 \\
45,0\end{array}$ & $\begin{array}{l}0,235 \\
0,288 \\
5,15 \\
4,44\end{array}$ & $\begin{array}{l}30,4 \\
14,2 \\
280 \\
153\end{array}$ & $\begin{array}{l}0,40 \\
0,17 \\
1,50 \\
1,45\end{array}$ & $\begin{array}{l}0,038 \\
0,055 \\
1,56 \\
1,36\end{array}$ & $\begin{array}{l}0,070 \\
0,073 \\
1,58 \\
1,42\end{array}$ & $\begin{array}{r}6,3 \\
4,1 \\
14,5 \\
9,9\end{array}$ & $\begin{array}{l}820 \\
200 \\
790 \\
340\end{array}$ & $\begin{array}{l}790 \\
180 \\
720 \\
290\end{array}$ & $\begin{array}{l}0,43^{*} \\
0,78 \\
4,4 \\
3,0\end{array}$ & $\begin{array}{c}\mathbf{1 5} \\
\mathbf{5 , 3} \\
3,3 \\
\mathbf{3 , 3}\end{array}$ \\
\hline$\Sigma$ & 3,98 & 4,63 & 37,7 & 45,0 & 94,3 & 10,1 & 478 & 3,52 & 3,01 & 3,14 & & & & & \\
\hline Lit. ${ }^{5}$ & & & & & & 14,0 & 481 & 3,07 & 3,43 & 3,58 & & & & & \\
\hline
\end{tabular}

Tab. 1. Edelgasgehalt und Mineralzusammensetzung der einzelnen Fraktionen. Die Ergebnisse für die Meteorite A k a b a, $\mathrm{Breitscheid,B} \mathrm{Buderheim,} \mathrm{Hayes} \mathrm{Center} \mathrm{und} \mathrm{Mocs} \mathrm{sind} \mathrm{bereits} \mathrm{in} \mathrm{einer} \mathrm{früheren} \mathrm{Arbeit}{ }^{9}$ enthalten. Zur Berechnung der in Spalte ${ }^{4} \mathrm{He}_{\mathrm{rad}}$. angeführten Werte wurde ein spallogenes Produktionsverhältnis ${ }^{4} \mathrm{He} /{ }^{3} \mathrm{He}=5$ angenommen. In der Spalte ${ }^{21} \mathrm{Ne}_{\mathrm{sp}}$. sind nur die Spallationsanteile der ${ }^{21} \mathrm{Ne}-$ Konzentrationen angeführt. Die mit ${ }^{*}$ bezeichneten ${ }^{21} \mathrm{Ne} \mathrm{sp}_{\mathrm{sp}}-\mathrm{K}_{\mathrm{K}}$ zentrationen in den $\mathrm{CuCl}_{2}-\mathrm{Phasen}$ wurden bezüglich der mit gelösten Silikatmengen korrigiert. Die Genauigkeit aller Angaben ist besser als $10 \%$, ausgenommen die Werte für ${ }^{21} \mathrm{Ne}_{\mathrm{sp}}$. im Nickeleisen und die daraus berechneten ${ }^{3} \mathrm{He} /{ }^{21} \mathrm{Ne}-\mathrm{Verh} a ̈ l t n i s s e$, wo Fehler bis zu 20\% und in Einzelfällen auch darüber auftreten können.

Tritium enthalten hätte. Von den erfolglosen Bemühungen, Tritium in Eisenmeteoriten zu messen ${ }^{15}, 18,19$, verdient die Arbeit von Bainbridge, SuEss und WäNKE besonderes Interesse. Um sicher zu sein, daß das Tritium aus Eisenmeteoriten nicht bei der Extraktion in der Metallschmelze zurückgehalten wird, wurde eine Probe des Eisenmeteoriten A r o o s im Sauerstoffstrom verbrannt. Auch in die-

18 E. L. Fireman u. J. DeFelice, Geochim. Cosmochim. Acta $18,183[1960]$. sem Versuch fanden sich nur maximal 5\% der zu erwartenden Tritiummenge. Man kann andererseits sicher sein, daß in der Regel der Großteil des Tritiums, das in Eisenmeteoriten während der Einwirkung der Höhenstrahlung gebildet wird, auch in ${ }^{3} \mathrm{He}$ zerfällt, da sonst die hohen ${ }^{3} \mathrm{He} /{ }^{4} \mathrm{He}-$ Verhältnisse (bis zu 0,32$)^{16}$ nicht erklärbar wären. Man nimmt daher allgemein an, daß das Tritium aus dem

19 A. E. Bainbridge, H. E. Suess u. H. Wänke, Geochim. Cosmochim. Acta 26, 471 [1962]. 
meteoritischen Metall erst nach dem Fall des Meteoriten auf die Erde verlorengeht. Sieht man von der hypothetischen Möglichkeit eines Einflusses der Feuchtigkeit ab, so bleibt eine Temperaturerhöhung als wahrscheinliche Ursache des Tritiumverlustes.

Die Aufbewahrungstemperatur eines Körpers auf der Erde kann mit ca. $300^{\circ} \mathrm{K}$ angenommen werden. Hingegen beträgt die Temperatur, die ein schwarzer Körper in der Erdbahn annehmen würde, nur $280^{\circ} \mathrm{K}$. Den Rechnungen Arnolds ${ }^{20,} 21$ zufolge, erlangen $30-40 \%$ aller Meteorite auf Grund ihrer Bahnen Temperaturen von mehr als $390^{\circ} \mathrm{K}$. Es wäre somit durchaus verständlich, daß die Meteorite teilweise bereits im Weltraum ihr Tritium verlieren. Wegen der geringen Halbwertszeit von Tritium müssen solche Meteorite, bei denen man aus einer Mindermenge von ${ }^{3} \mathrm{He}$ in der Metallphase auf einen Tritiumverlust schließt, während ihrer gesamten Lebensdauer als kleine Körper auf Bahnen gewesen sein, auf denen die für diese Verluste erforderlichen Temperaturen erreicht werden.

Wie Abb. 1 zeigt, geht der Tritiumverlust im Nickeleisen im allgemeinen mit dem ${ }^{3} \mathrm{He}$-Verlust im Pyroxen parallel. Es gibt jedoch Meteorite, die beträchtliche Mindermengen von ${ }^{3} \mathrm{He}$ im Nickeleisen aufweisen, hingegen im Pyroxen keinen oder nur geringen ${ }^{3} \mathrm{He}-$ Verlust gegenüber dem Olivin zeigen. Ein solcher Fall ist B a th. Tritium geht aus der Metallphase demnach bereits bei Temperaturen verloren, bei denen in den Nichtmetallkomponenten noch keine Verluste von ${ }^{3} \mathrm{He}$ zu bemerken sind.

Noch empfindlicher als Pyroxen ist der Troilit gegenüber Diffusionsverlusten. Für den Troilit sind die ${ }^{3} \mathrm{He}-K o n z e n t r a t i o n e n$ fast in allen Meteoriten sehr stark erniedrigt bzw. ganz verschwunden. Bei der Betrachtung der Tab. 1 ist daran zu erinnern, daß die Bromphase nie reinen Troilit lieferte, sondern besonders bei den weiter zurückliegenden Versuchen stets auch Olivin zum Teil in beträchtlichen Mengen mit aufgeschlossen wurde. Durch eine Verbesserung der Versuchstechnik konnte bei den späteren Versuchen der Olivinanteil beträchtlich herabgesetzt werden.

In Meteoriten mit einem hohen Verlust an ${ }^{3} \mathrm{He}$ im Pyroxen aber geringen Fehlmengen im Nickeleisen stets bezogen auf den Olivin - kann die Temperaturerhöhung erst zu einem Zeitpunkt eingetreten sein,

20 J. R. Arnold, Astrophys. J. 141, 1536 [1965]. als bereits der Großteil des ${ }^{3} \mathrm{He}$ gebildet war. Einen Fall dieser Art haben wir im Meteoriten Breit s cheid. In Abschnitt 2 b werden wir einen weiteren Hinweis auf eine derartige Temperaturgeschichte dieses Meteoriten kennenlernen.

Unter den 10 Hypersthen-Chondriten zeigt nur Mocs einen starken, beinahe 100-proz. Tritiumverlust. Bronzit-Chondrite mit hohem Tritiumverlust sind: Bath, Cullis on und Texline; alle drei besitzen niedrige Bestrahlungsalter. Beide Bronzit-Chondrite mit hohen Bestrahlungsaltern Breits cheid und R os e City - zeigen keinen oder nur sehr geringen Verlust von Tritium. Bei den Hypersthen-Chondriten scheinen jedoch Meteorite mit kleinen Bestrahlungsaltern die geringeren Tritiumverluste erlitten zu haben. Es ist noch nicht möglich, hieraus irgendwelche weitere Schlüsse zu ziehen. Dazu ist die Zahl der untersuchten Meteorite zu gering, auch könnten die Diffusionseigenschaften für Tritium im Nickeleisen für die beiden Meteoritklassen wegen des unterschiedlichen Nickelgehaltes verschieden sein.

\section{b) Radiogenes ${ }^{4} \mathrm{He}$}

Wir haben uns im Rahmen dieser Untersuchungen vor allem für solche Meteorite interessiert, bei denen vermutlich Verluste sowohl von spallogenem ${ }^{3} \mathrm{He}$ als auch von radiogenem ${ }^{4} \mathrm{He}$ auftraten. Es wurden jedoch drei Meteorite aufgenommen, von denen wir sicher sein konnten, daß sie keine oder nur sehr geringe Verluste von radiogenem Helium erlitten haben. Es sind dies $\mathrm{Holbrook}, \mathrm{Bath}$ und $\mathrm{Ochansk}$. Alle drei Meteorite haben radiogene Heliumalter von nahe 4 Milliarden Jahren (siehe Tab. 2). Wie aus den Meßdaten dieser Meteorite hervorgeht, liegen die radiogenen Heliumkonzentrationen im Olivin bei ca. $1000 \cdot 10^{-8} \mathrm{~cm}^{3} \mathrm{He} / \mathrm{g}$ und im Pyroxen bei ca. $2000 \cdot 10^{-8} \mathrm{~cm}^{3} \mathrm{He} / \mathrm{g}$. Der radiogene Heliumgehalt des Pyroxens ist dabei stets etwa doppelt so hoch wie der des Olivins. Es war besonders wichtig, diese Anfangsbedingungen zu kennen, denn nur so lassen sich Angaben über den Verlust von radiogenem Helium machen.

Analog zu den Ergebnissen für ${ }^{3} \mathrm{He}$ erwiesen sich die Verluste von radiogenem Helium im Pyroxen als wesentlich größer als im Olivin. Der Meteorit Cullis on enthält im Pyroxen nur mehr knapp $10 \%$ des ursprünglichen Heliumgehaltes, hingegen ist im Olivin noch kein deutlicher Heliumverlust zu

21 J. R. Annold, Astrophys. J. 141, 1548 [1965]. 

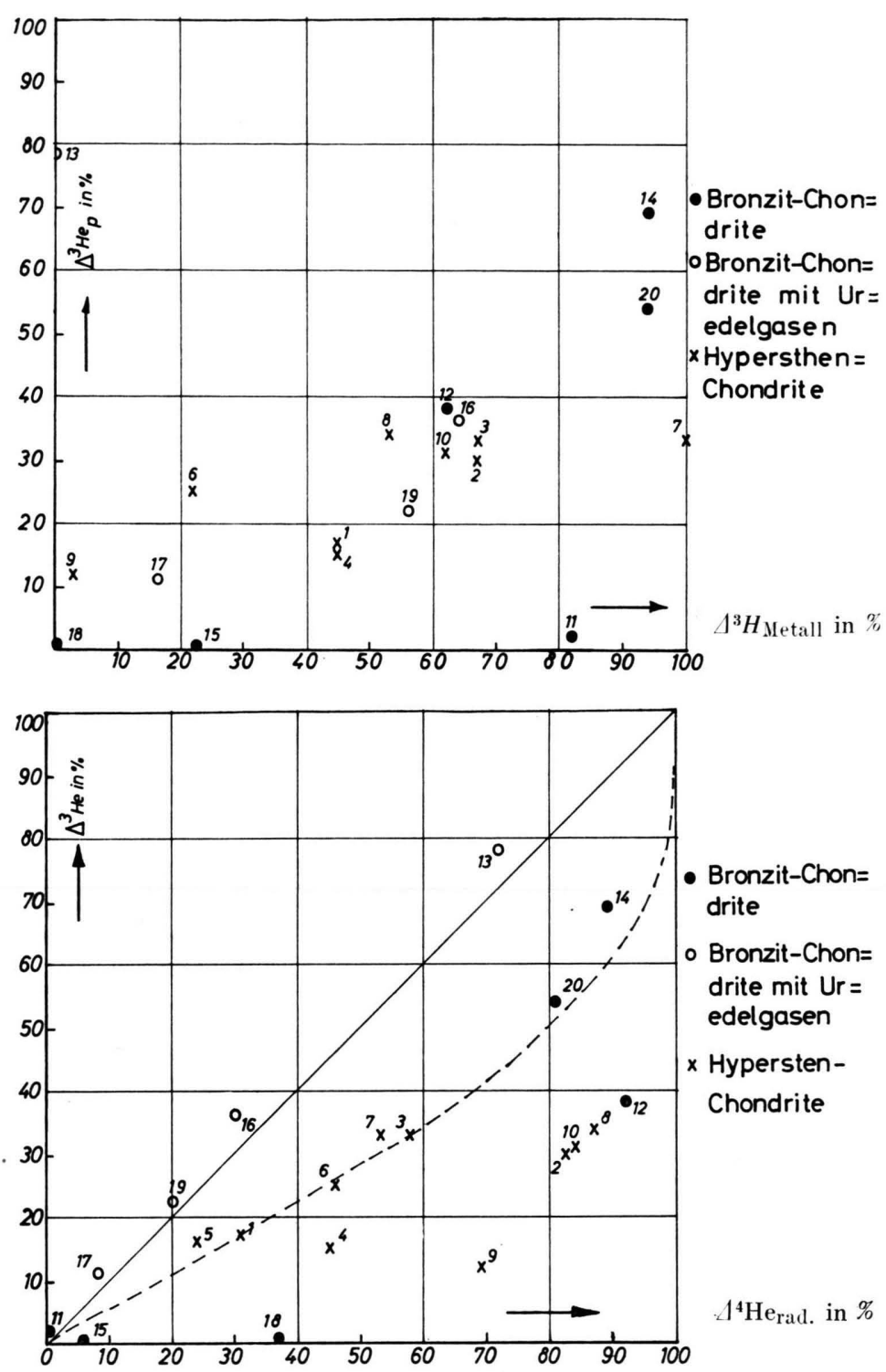

merken. In allen Fällen, in denen der ${ }^{4} \mathrm{He}$-Gehalt des Olivins erniedrigt ist, muß es sich also um sehr drastische Temperaturerhöhungen gehandelt haben. Eine Erwärmung auf Grund einer näher an die Sonne heranführenden Bahn ist für diese Fälle wohl ausgeschlossen.

Die Verteilung des radiogenen Heliums zwischen Olivin und Pyroxen - der Unterschied wird noch deutlicher, wenn man Kristalle ab $30 \mu$ aufwärts berücksichtigt $^{22}$, - legt den Schluß nahe, daß der Großteil des Urans und Thoriums im Pyroxen, oder besser gesagt, in unserem unlöslichen Rückstand
Bronzit-Chon= drite

Bronzit-Chon= drite mit Ur= edelgasen

HyperstenChondrite

$1^{4} \mathrm{He}$ rad. in $\%$ Innere.
Abb. 1. Prozentualer Verlust von Tritium im Nickeleisen und von ${ }^{3} \mathrm{He}$ im Pyroxen. Ein Trend zur Proportionalität ist deutlich erkennbar. Die Zahlen beziehen sich auf die Tab. 2 und 3.
Abb. 2. Prozentualer Verlust von ${ }^{3} \mathrm{He}$ und ${ }^{4} \mathrm{He}_{\text {rad. im Pyroxen. Alle Werte sind auf }}$ Olivin bezogen unter der Annahme, daß Diffusionsverluste im Olivin nicht aufgetreten sind. Die gestrichelte Kurve gibt die theoretische Abhängigkeit der Diffusionsverluste von ${ }^{3} \mathrm{He}$ und ${ }^{4} \mathrm{He}_{\mathrm{rad}}$. wieder. Hierbei wurde angenommen, daß die für die Diffusionsverluste verantwortliche Erwärmung während der Einwirkungsdauer der Höhenstrahlung gleich hoch war und Diffusion

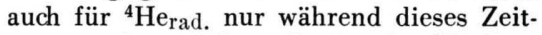
raums auftrat, daß am Beginn der ${ }^{3} \mathrm{He}$-Produktion bereits alles ${ }^{4} \mathrm{He}_{\mathrm{rad}}$. vorhanden war und daß alle Pyroxenkörner kugelförmig und von einheitlicher Korngröße sind. Die Zahlen beziehen sich auf die Tab. 2 und 3.

22 H. Hintenberger, H. König, L. Schultz, H. Wänke u. F. W lotzka, Z. Naturforschg. 19 a, 88 [1964].

(Pyroxen und Feldspäte) sitzt, der Olivin jedoch nahezu frei von diesen Radioelementen ist. Die Oberflächenschichten des Olivins enthalten häufig auch wesentlich höhere Heliumkonzentrationen als das

Dieses zeigt sich in den manchmal recht hohen ${ }^{4}$ He-Mengen der Bromphasen, in denen stets auch gewisse Mengen Olivin gelöst werden (hauptsächlich natürlich Oberflächenmaterial).

Selbst das metallische Nickeleisen enthält ${ }^{4} \mathrm{He}$ nicht spallogenen Ursprungs, und zwar fanden wir stets Werte um $200 \cdot 10^{-8} \mathrm{~cm}^{3} / \mathrm{g}$; ausgenommen solche 


\begin{tabular}{|c|c|c|c|c|c|c|c|c|c|c|c|c|}
\hline & Meteorit & $\frac{{ }^{3} \mathrm{He}_{\mathrm{P}} .}{{ }^{3} \mathrm{He}_{0}}$ & $\begin{array}{l}{ }^{3} \mathrm{He}_{\mathrm{P}} . \\
\text { in } \%\end{array}$ & $\begin{array}{c}{ }^{4} \mathrm{He}_{\text {rado }} \\
\text { in } \\
10^{-8} \\
\mathrm{~cm}^{3} / \mathrm{g}\end{array}$ & $\begin{array}{c}{ }^{4} \mathrm{He}_{\text {radp }} \\
\text { in } \\
10^{-8} \\
\mathrm{~cm}^{3} / \mathrm{g}\end{array}$ & $\frac{{ }^{4} \mathrm{He}_{\mathrm{rado}}}{{ }^{4} \mathrm{He}_{\mathrm{radP}}}$ & $\begin{array}{c}{ }^{4} \mathrm{He}_{\mathrm{radP}} \\
\text { in } \%\end{array}$ & $\begin{array}{r}{ }^{4} \mathrm{He}_{\text {rado }} \\
\text { Korr. I } \\
\text { in }\end{array}$ & $\left|\begin{array}{c}{ }^{4} \mathrm{He}_{\text {radP }} \\
\text { Korr. II } \\
10^{-8} \mathrm{~cm}^{3} \mid\end{array}\right|$ & $\begin{array}{c}{ }^{4} \mathrm{He}_{\text {radm }} \\
\text { Korr. }\end{array}$ & $\begin{array}{c}\text { U-Th-He } \\
\text { Alter } \\
\text { in } \\
10^{9} \mathrm{Ja}\end{array}$ & $\begin{array}{l}\mathrm{K}-\mathrm{Ar} \\
\text { Alter } \\
\text { in } \\
\text { hren }\end{array}$ \\
\hline \multicolumn{13}{|c|}{ Hypersthen-Chondrite } \\
\hline 1 & Akaba & 0,83 & 17 & 570 & 790 & 0,69 & 31 & 950 & 1250 & 160 & $2,98^{4}$ & $3,6 \quad 26$ \\
\hline 2 & Bruderheim & 0,70 & 30 & 380 & 130 & 0,17 & 83 & 180 & 310 & 130 & $1,27^{5}$ & $1,84^{3}$ \\
\hline 3 & Goodland & 0,67 & 33 & 130 & 110 & 0,42 & 58 & 160 & 300 & 70 & $0,50^{5}$ & $0,94^{3}$ \\
\hline 4 & Hayes Center & 0,85 & 15 & 100 & 110 & 0,55 & 45 & 130 & 160 & 0 & $0,78^{4}$ & $0,65^{3}$ \\
\hline 5 & Holbrook & 0,84 & 16 & 1140 & 1740 & 0,76 & 24 & 2100 & 2700 & - & $4,10^{4}$ & $4,44^{3}$ \\
\hline 6 & Kiel & 0,75 & 25 & 870 & 940 & 0,54 & 46 & 1250 & 1900 & 480 & $3,37^{5}$ & $4,26^{3}$ \\
\hline 7 & Mocs & 0,67 & 33 & 580 & 540 & 0,47 & 53 & 810 & 1450 & 220 & $2,37^{4}$ & $3,8 \quad 26$ \\
\hline 8 & New Concord & 0,66 & 34 & 390 & 100 & 0,13 & 87 & 150 & 280 & 240 & $0,92^{5}$ & $2,06^{3}$ \\
\hline 9 & Parnallee & 0,88 & 12 & 670 & 420 & 0,31 & 69 & 480 & 570 & 290 & $2,35^{5}$ & - \\
\hline \multirow[t]{2}{*}{10} & Walters & 0,69 & 31 & 320 & 100 & 0,16 & 84 & 150 & 250 & 170 & $0,91^{5}$ & 2,7727 \\
\hline & \multicolumn{12}{|c|}{ Bronzit-Chondrite } \\
\hline 11 & Bath & 0,98 & 2 & 1840 & 1760 & 1,05 & -5 & 1800 & 1800 & 170 & 3,66 & - \\
\hline 12 & Beddgelert & 0,62 & 38 & 490 & 80 & 0,08 & 92 & 130 & 270 & 120 & $0,42^{4}$ & $2,8 \quad 26$ \\
\hline 13 & Breitscheid & 0,22 & 78 & 840 & 470 & 0,28 & 72 & 2100 & 47000 & 320 & $1,35^{4}$ & $3,3 \quad 26$ \\
\hline 14 & Cullison & 0,31 & 69 & 800 & 174 & 0,11 & 89 & 560 & 3500 & 162 & $1,11^{5}$ & - \\
\hline 15 & Ochansk & 1,01 & -1 & 1000 & 1880 & 0,94 & 6 & 1900 & 1900 & 220 & $4,53^{5}$ & $3,70^{3}$ \\
\hline 16 & Pantar & 0,64 & 36 & 1020 & 1430 & 0,70 & 30 & 2200 & 4500 & 170 & $3,49^{5}$ & 3,83 \\
\hline 17 & Pultusk & 0,89 & 11 & 930 & 1680 & 0,92 & 8 & 1900 & 2300 & 310 & $3,87^{5}$ & $3,93^{3}$ \\
\hline 18 & Rose City & 1,33 & -33 & 80 & 100 & 0,63 & 37 & 80 & 80 & 55 & $0.54^{6}$ & $1,03^{3}$ \\
\hline 19 & Tabor & 0,78 & 22 & 1110 & 1780 & 0,80 & 20 & 2300 & 3300 & 170 & $3,64^{5}$ & - \\
\hline \multirow[t]{2}{*}{20} & Texline & 0,46 & 54 & 720 & 280 & 0,19 & 81 & 610 & 1900 & 100 & $1,33^{5}$ & - \\
\hline & \multicolumn{12}{|c|}{ Amphoter. Chondrit } \\
\hline 21 & Dhurmsala & 0,68 & 32 & 720 & 290 & - & - & - & - & 760 & $1,60^{5}$ & - \\
\hline
\end{tabular}

Tab. 2. Konzentrationen und Verhältnisse von ${ }^{3} \mathrm{He}$ und ${ }^{4} \mathrm{He}_{\mathrm{rad}}$. in den verschiedenen Mineralkomponenten der Meteorite sowie die prozentualen Diffusionsverluste von spallogenem $\left({ }^{3} \mathrm{He}\right)$ und radiogenem $\left({ }^{4} \mathrm{He}_{\mathrm{rad}}\right.$.) Helium im Pyroxen. Die angegebenen Alter sind Literaturwerte an Gesamtproben $(\mathrm{M}=$ Metall, $\mathrm{O}=$ Olivin, $\mathrm{P}=$ Pyroxen $)$.

Meteorite, die wie Goodland, Hayes Center, Beddgelert und Rose City sehr starken Erwärmungen ausgesetzt waren. Das ist auch aus dem stark verminderten radiogenen Heliumgehalt des Olivins ersichtlich. Bei diesen ${ }^{4} \mathrm{He}-\mathrm{Mengen}$ im Nickeleisen handelt es sich zumindest teilweise um eingeschossene $\alpha$-Teilchen radiogenen Ursprungs. Gelegentliche Abweichungen von diesem ${ }^{4} \mathrm{He}$-Gehalt sind sowohl durch Korngrößenunterschiede als auch durch eine eventuelle Beimischung von Urhelium zu erklären.

Auf Grund der im Abschnitt 2 a diskutierten ${ }^{3} \mathrm{He}-$ Verluste im Nickeleisen (bedingt durch Tritiumverluste) werden auch die in Tab. 1 angegebenen Konzentrationen von radiogenem Helium korrekturbedürftig. Tab. 2 enthält diese Werte korrigiert auf Tritiumverlust. Wiederum wurde zur Berechnung des Anteils von ${ }^{4} \mathrm{He}_{\text {rad. }}$ ein spallogenes ${ }^{4} \mathrm{He} /{ }^{3} \mathrm{He}$-Verhältnis von 4 angenommen. Die Verschiebungen durch diese Korrektur sind jedoch gering. Eine Ausnahme 26 H. WänKe u. H. KönIG, Z. Naturforschg. 14 a, 860 [1959]. bildet der Meteorit $\mathrm{H}$ a y e s $\mathrm{C}$ e $\mathrm{n}$ t e r, bei dem kein radiogener Heliumanteil im Nickeleisen übrigbleibt.

In Abb. 2 haben wir die Verluste von ${ }^{3} \mathrm{He}$ und ${ }^{4} \mathrm{He}_{\mathrm{rad}}$ im Pyroxen aller untersuchten Meteorite eingetragen. Falls die für die Diffusionsverluste entscheidende Temperatur auf Grund einer bestimmten Bahn des Meteoriten zustandekam, und keine Bahnänderung stattfand, dann muß die Diffusion während der ganzen Einwirkungsdauer der Höhenstrahlung gleichmäßig vor sich gegangen sein. Zu dem Zeitpunkt, als die Produktion von ${ }^{3} \mathrm{He}$ auf Grund der Einwirkung der Höhenstrahlung begann, war schon fast alles ${ }^{4} \mathrm{He}_{\mathrm{rad}}$. vorhanden. Die relativen Verluste des radiogenen Heliums müßten somit größer sein als die von ${ }^{3} \mathrm{He}$. Die Meßpunkte sollten daher nahe dieser Kurve liegen. Dieses ist nur für einige Meteorite der Fall. Die Verluste an radiogenem Helium in Abb. 2 sind stets nur bezogen auf den radiogenen ${ }^{4} \mathrm{He}-$ Gehalt im Olivin. Für die Mehrzahl der untersuchten Hypersthen-Chondrite sind die tat27 P. Eberhardt, O. Eugster, J. Geiss u. K. Marti, Vorabdruck November 1965. 
sächlichen Verluste an radiogenem Helium im Pyroxen noch wesentlich größer, da häufig auch der Olivin Heliumverluste erlitten hat. Von den 10 Hypersthen-Chondriten kämen damit 7 unter der gestrichelten Kurve zu liegen. Die Verluste von ${ }^{4} \mathrm{He}_{\mathrm{rad}}$. müssen hier also bereits im Mutterkörper des Meteoriten stattgefunden haben. Eine Ausnahme bilden die Meteorite $\mathrm{Holbrook}, \mathrm{Ki}$ el und möglicherweise $\mathrm{Akaba}$.

Die Punkte für die Bronzit-Chondrite liegen meist oberhalb der theoretischen Kurve, vor allem die der uredelgashaltigen Meteorite B reits c heid, Pan $\mathrm{tar}, \mathrm{T} a \mathrm{~b}$ or und Pultusk. Alle diese Meteorite zeigen einen fast identischen relativen ${ }^{3} \mathrm{He}$ - und

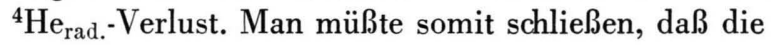
für die Diffusion verantwortliche Erwärmung erst eingetreten ist, als der überwiegende Teil nicht nur des radiogenen Heliums, sondern auch des spallogenen Heliums bereits gebildet war.

In unserer ersten Arbeit ${ }^{9}$ hatten wir fälschlicherweise einen gleich großen radiogenen Heliumgehalt für Olivin und Pyroxen angenommen. (Meßdaten an Meteoriten ohne Diffusionsverluste standen uns damals noch nicht zur Verfügung.) Wir hatten deshalb für Breitscheid im Pyroxen auf einen höheren relativen Verlust von ${ }^{3} \mathrm{He}$ als von ${ }^{4} \mathrm{He}_{\mathrm{rad}}$. geschlossen. In Wahrheit erwiesen sich diese Verluste als fast genau gleich groß. Unser damaliger Schluß hinsichtlich einer frühen Einwirkung der Höhenstrahlung ist daher nicht mehr so zwingend. Eine durch eine Bahnänderung bedingte Erwärmung am Ende des durch das Bestrahlungsalter gegebenen Zeitintervalls würde das gleiche Ergebnis liefern. Sehr wahrscheinlich erscheint diese Erklärung jedoch nicht.

Schon im Abschnitt 2 a hatten wir für B reit scheid auf Grund eines Fehlens des Tritiumverlustes geschlossen, daß für diesen Meteorit die entscheidende Temperaturerhöhung erst eingetreten sein kann, nachdem bereits das meiste ${ }^{3} \mathrm{He}$ gebildet war. Eine frühe Einwirkung der Höhenstrahlung an der Oberfläche des Meteoritenmutterkörpers bei relativ tiefer Temperatur würde die gefundenen Tatsachen zwanglos erklären und die Annahme einer Bahnänderung kurz vor dem Aufschlag auf die Erde unnötig machen.

Es wäre denkbar, daß die Gleichheit der relativen Diffusionsverluste von ${ }^{3} \mathrm{He}$ und ${ }^{4} \mathrm{He}_{\mathrm{rad}}$. auf Grund eines Korngrößeneffektes zustandekommt. Alle Körner unterhalb eines bestimmten Durchmessers könn- ten praktisch leergelaufen sein, und zwar sowohl für das radiogene wie auch das spallogene Helium, während ein Rest von Körnern mit größeren Durchmessern praktisch keinerlei Diffusionsverluste aufweist; also alles radiogene und spallogene Helium in diesen enthalten ist. Auch eine in gewissem Maße zu erwartende ungleichmäßige Verteilung der ursprünglichen Konzentration von radiogenem Helium könnte von Einfluß sein. (Pyroxenkörner, die klein gegenüber der Reichweite der $\alpha$-Teilchen sind, enthalten sicher weniger radiogenes Helium als solche, bei denen der Korndurchmesser die Reichweite der $\alpha$-Teilchen übersteigt. Das spallogene Helium hingegen ist über alle Körner homogen verteilt.) Für einen Korngrößeneffekt sprechen auch die merklichen Verluste von ${ }^{21} \mathrm{Ne}$ im Pyroxen.

Beide zuletzt angeführten Punkte sollen in zukünftigen Diffusionsexperimenten geklärt werden. Mit Sicherheit können wir im Augenblick nur sagen, daß die für den Verlust von radiogenem Helium verantwortliche Erwärmung bei den Bronzit-Chondriten in den meisten Fällen in einer sonnennäheren Bahn zu suchen ist. Auch für die Hypersthen-Chondrite sind die auf diese Weise entstandenen Verluste von radiogenem Helium nicht vernachlässigbar. Hier finden sich auch zahlreiche Fälle, bei denen starke Verluste von radiogenem Helium durch eine Erwärmung im Meteoritenmutterkörper zustandegekommen sind.

Wir können noch nicht sagen, ob die Gleichheit der relativen Verluste von spallogenem und radiogenem Helium auf die Bronzit-Chondrite bzw. auf die uredelgashaltigen Bronzit-Chondrite beschränkt ist, und die Hypersthen-Chondrite tatsächlich ein anderes Verhalten zeigen. Bei den letzteren kommen zu den Diffusionsverlusten von radiogenem Helium während der Einwirkung der Höhenstrahlung in vielen Fällen noch die zeitlich früher liegenden Verluste im Meteoritenmutterkörper hinzu, wodurch sich das gesamte Bild wesentlich verwischt.

Anders ${ }^{23}$ hat als erster aus den Edelgasdaten von Hypersthen-Chondriten auf eine starke Erwärmung des Meteoritenmutterkörpers vor etwa 400 Millionen Jahren geschlossen. Besonders günstig für die Datierung dieses Zeitpunktes ist das radiogene Helium im Pyroxen. In Tab. 2 sind die auf Diffusionsverluste während der Einwirkungsdauer der Höhenstrahlung korrigierten ${ }^{4} \mathrm{He}_{\mathrm{rad}}$. Werte angegeben. Die Meßwerte wurden wiederum auf Grund der Mindermengen von ${ }^{3} \mathrm{He}$ im Pyroxen gegenüber dem Olivin korrigiert.

23 E. Anders, Space Sci. Rev. 3, 583 [1964ך. 
Die Korrektur erfolgte einmal auf Grund der ausgezogenen Geraden in Abb. 2 (Korrektur I) und einmal auf Grund der gestrichelten Kurve (Korrektur II). Fünf der untersuchten Hypersthen-Chondrite zeigen bei Korrektur nach I einen radiogenen Heliumgehalt im Pyroxen von nahe um $160 \cdot 10^{-8}$ $\mathrm{cm}^{3} \mathrm{He} / \mathrm{g}$. Ein Gehalt an ${ }^{4} \mathrm{He}_{\mathrm{rad}}$. von $2000 \cdot 10^{-8} \mathrm{~cm}^{3}$ entspricht einem Alter von etwa $4,5 \cdot 10^{9} \mathrm{Jahren}$, für $160 \cdot 10^{-8} \mathrm{~cm}^{3}{ }^{4} \mathrm{He}_{\mathrm{rad}} / \mathrm{g}$ errechnet sich somit ein Alter von $560 \cdot 10^{6} \mathrm{Jahren}$. Dieses ist eine Bestätigung der Hypothese von Anders ${ }^{21}$. Aus dem Gehalt von radiogenem Helium im gesamten Meteoriten ergeben sich für Bruderheim, New Concord und W alters wesentlich höhere Alter. Eine Korrektur nach II ergäbe beinahe doppelt so hohe Heliumgehalte, jedoch wesentlich größere Streuungen.

Sicher ist eine Erwärmung im Meteoritenmutterkörper vor ca. 500 Millionen Jahren, wie von ANDERs ${ }^{23}$ vorgeschlagen, eine gute Erklärung für diese tiefen radiogenen Alter.

Sie ist aber nicht die einzige Erklärung. Die $160 \cdot 10^{-8} \mathrm{~cm}^{3}{ }^{4} \mathrm{He}_{\mathrm{rad}} / \mathrm{g}$ entsprechen $8 \%$ der Menge wie wir sie in den Meteoriten ohne sekundäre Erwärmung fanden. In einem abgeschlossenen System würden diese $8 \%$ in einem Meteoriten mit einem Porenvolumen von 5\% einer Löslichkeit von Helium im Pyroxen von 0,4\% entsprechen. Die $8 \%$ Helium im Pyroxen würden dann den unteren Grenzwert darstellen, bis zu welchem die Heliumkonzentration vermindert werden kann. Bemerkenswert hierzu ist die Tatsache, daß die Bronzit-Chondrite B ed d g e lert und $\mathrm{R}$ o se $\mathrm{C}$ it y einen ähnlichen Gehalt von radiogenem Helium im Pyroxen aufweisen.

$$
\text { c) }{ }^{21} \mathrm{Ne} \text { und das }{ }^{3} \mathrm{He} /{ }^{21} \mathrm{Ne} \text {-Verhältnis }
$$

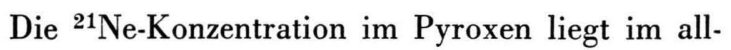
gemeinen unter der des Olivins. Die Ursache ist auch hier teilweise ein Diffusionsverlust. Generell sind jedoch im Pyroxen etwas tiefere ${ }^{21} \mathrm{Ne}-K o n z e n t r a-$ tionen zu erwarten als im Olivin, und zwar auf Grund der chemischen Zusammensetzung. Olivin enthält etwa 23\% Magnesium und etwa $18 \%$ Silicium, Pyroxen etwa 17\% Magnesium und 25\% Silicium. Hintenberger, König, Schultz, Wänke und WLotzKa ${ }^{22}$ fanden für den Meteoriten $\mathrm{H} \mathrm{olb} \mathrm{r} \mathrm{o} \mathrm{ok}$ für das Verhältnis der Produktionsquerschnitte für ${ }^{21} \mathrm{Ne}$ aus $\mathrm{Mg}$ und $\mathrm{Si}$ einen Wert von 1,8 $\pm 0,4$. Jedoch

24 P. Signer u. A. O. Nier, Researches on Meteorites, Ed. C. B. Moore, pp. 7-35, John Wiley \& Sons, New York 1962. ist dieser Wert sicherlich etwas von der Strahlungshärte abhängig.

Auf die großen Schwankungen der ${ }^{3} \mathrm{He} /{ }^{21} \mathrm{Ne}-\mathrm{Ver}$ hältnisse in Chondriten wurde schon oft hingewiesen. Von besonderem Interesse waren für uns Messungen an Meteoriten mit extremen ${ }^{3} \mathrm{He} /{ }^{21} \mathrm{Ne}-$ Verhältnissen. Natürlich bedingen Diffusionsverluste, die vor allem das ${ }^{3} \mathrm{He}$ betreffen, eine Verringerung dieses Verhältnisses. Im Olivin sind jedoch Diffusionsverluste der spallogenen Edelgase, von Ausnahmefällen abgesehen, zu vernachlässigen. $\mathrm{Akaba}$ und $\mathrm{Ki}$ el mit extrem hohen ${ }^{3} \mathrm{He} /{ }^{21} \mathrm{Ne}-$ Werten zeigen ebenso wie $\mathrm{Parnallee}$ mit einem extrem tiefen Wert die gleichen ${ }^{3} \mathrm{He} /{ }^{21} \mathrm{Ne}-$ Verhältnisse im Olivin und Pyroxen. Im letzteren Falle ist eine Erniedrigung durch Diffusion auszuschließen. Trotz extremer Unter-

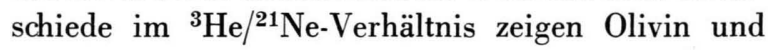
Pyroxen recht ähnliche Isotopenverhältnisse. Reine $(\mathrm{n}, \alpha)$-Prozesse am Magnesium, durch die kein ${ }^{20} \mathrm{Ne}$ entstehen würde, können daher nur eine untergeordnete Rolle spielen.

Ein sehr tiefes ${ }^{3} \mathrm{He} /{ }^{21} \mathrm{Ne}-$ Verhältnis fanden wir auch für Walters $(=2,4$ im Olivin) aber auch für den Bronzit-Chondriten Cullis on (= 1,9 im Olivin). Cullis on zeigt allerdings im Pyroxen eine starke Erniedrigung dieses Verhältnisses auf Grund eines ${ }^{3} \mathrm{He}-$ Verlustes. Wegen des hohen Gehaltes von radiogenem Helium im Olivin von $\mathrm{Cu}$ llis on lassen sich Diffusionsverluste in dieser Mineralkomponente wohl ausschließen.

Variationen des ${ }^{3} \mathrm{He} /{ }^{21} \mathrm{Ne}-$ Verhältnisses um mehr als einen Faktor 4 (Olivin $\mathrm{Kiel}=8,2$, Olivin Cullison $=1,9$ ) sind also zuverlässig nachgewiesen.

Wie wir in Abschnitt 2 a ausgeführt haben, sind die im Nickeleisen der Chondrite zu erwartenden ${ }^{3} \mathrm{He}-K o n z e n t r a t i o n e n$ unabhängig von der Korngröße. Korrigiert man die ${ }^{3} \mathrm{He}-M e n g e n$ im Nickeleisen hinsichtlich des Tritiumverlustes und berechnet

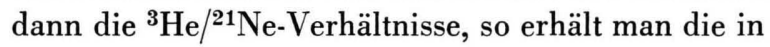
Tab. 3, letzte Spalte, aufgeführten Werte. Aus Messungen an Eisenmeteoriten wissen wir, da $\beta$ dieses Verhältnis zwischen 70 und 100 liegen müßte ${ }^{16,24,25}$. Für Eisenmeteorite mit kleiner Masse gilt ein Verhältnis von 70, und dieser Wert nimmt für große Meteorite mit steigendem Abstand von der Meteoritenoberfläche zu.

25 M. E. Lipschutz, P. Signer u. E. Anders, J. Geophys. Res. 70, 1473 [1965]. 


\begin{tabular}{|c|c|c|c|c|c|c|c|c|}
\hline & Meteorit & $\begin{array}{c}{ }^{21 \mathrm{Ne}} \\
\text { in } \\
10^{-8} \mathrm{~cm}^{3} / \mathrm{g}\end{array}$ & $\frac{{ }^{3} \mathrm{He}_{0}}{{ }^{21} \mathrm{Ne}_{0}}$ & $\frac{{ }^{3} \mathrm{He}_{\mathrm{M}}}{{ }^{3} \mathrm{He}_{0}}$ & $\begin{array}{l}{ }^{3} \mathrm{H}_{\mathrm{M}} \\
\text { in } \%\end{array}$ & $\frac{{ }^{3} \mathrm{He}_{\mathrm{P}}}{{ }^{3} \mathrm{He}_{0}}$ & $\begin{array}{l}{ }^{3} \mathrm{He}_{\mathrm{P}} \\
\text { in } \%\end{array}$ & $\frac{{ }^{3} \mathrm{He}_{\mathrm{M} \text { korr }}}{{ }^{21} \mathrm{Ne}_{\mathrm{M}}}$ \\
\hline \multicolumn{6}{|c|}{ Hypersthen-Chondrite } & \multicolumn{3}{|c|}{$\cdot$} \\
\hline $\begin{array}{r}1 \\
2 \\
3 \\
4 \\
5 \\
6 \\
7 \\
8 \\
9 \\
10\end{array}$ & $\begin{array}{l}\text { Akaba } \\
\text { Bruderheim } \\
\text { Goodland } \\
\text { Hayes Center } \\
\text { Holbrook } \\
\text { Kiel } \\
\text { Mocs } \\
\text { New Concord } \\
\text { Parnallee } \\
\text { Walters }\end{array}$ & $\begin{array}{l}5,11 \\
7,43 \\
4,44 \\
9,72 \\
5,36 \\
2,45 \\
4,82 \\
1,06 \\
1,65 \\
1,47\end{array}$ & $\begin{array}{l}7,0 \\
4,9 \\
4,8 \\
3,7 \\
3,8 \\
8,2 \\
4,6 \\
3,4 \\
2,0 \\
2,4\end{array}$ & $\begin{array}{c}0,58 \\
0,50 \\
0,50 \\
0,58 \\
- \\
0,68 \\
0,37 \\
0,55 \\
0,74 \\
0,52\end{array}$ & $\begin{array}{r}45 \\
67 \\
67 \\
45 \\
- \\
22 \\
100 \\
53 \\
3 \\
62\end{array}$ & $\begin{array}{l}0,83 \\
0,70 \\
0,67 \\
0,85 \\
0,84 \\
0,75 \\
0,67 \\
0,66 \\
0,88 \\
0,69\end{array}$ & $\begin{array}{l}17 \\
30 \\
33 \\
15 \\
16 \\
25 \\
33 \\
34 \\
12 \\
31\end{array}$ & $\begin{array}{r}109 \\
56 \\
83 \\
56 \\
59 \\
41 \\
80 \\
39 \\
41 \\
23\end{array}$ \\
\hline \multicolumn{9}{|c|}{ Bronzit-Chondrite } \\
\hline $\begin{array}{l}11 \\
12 \\
13 \\
14 \\
15 \\
16 \\
17 \\
18 \\
19 \\
20\end{array}$ & $\begin{array}{l}\text { Bath } \\
\text { Beddgelert } \\
\text { Breitscheid } \\
\text { Cullison } \\
\text { Ochansk } \\
\text { Pantar } \\
\text { Pultusk } \\
\text { Rose City } \\
\text { Tabor } \\
\text { Texline }\end{array}$ & $\begin{array}{l}2,14 \\
1,43 \\
7,82 \\
0,66 \\
2,30 \\
1,07 \\
1,91 \\
8,93 \\
2,21 \\
2,44\end{array}$ & $\begin{array}{l}3,8 \\
4,2 \\
4,1 \\
1,9 \\
3,8 \\
3,2 \\
4,0 \\
3,0 \\
3,4 \\
3,7\end{array}$ & $\begin{array}{l}0,44 \\
0,52 \\
0,75 \\
0,40 \\
0,68 \\
0,51 \\
0,69 \\
0,78 \\
0,54 \\
0,40\end{array}$ & $\begin{array}{r}82 \\
62 \\
0 \\
94 \\
22 \\
64 \\
16 \\
-8 \\
56 \\
94\end{array}$ & $\begin{array}{l}0,98 \\
0,62 \\
0,22 \\
0,31 \\
1,01 \\
0,64 \\
0,89 \\
1,33 \\
0,78 \\
0,46\end{array}$ & $\begin{array}{r}2 \\
38 \\
78 \\
69 \\
-1 \\
36 \\
11 \\
-33 \\
22 \\
54\end{array}$ & $\begin{array}{l}29 \\
52 \\
45 \\
47 \\
65 \\
35 \\
19 \\
69 \\
75 \\
61\end{array}$ \\
\hline \multicolumn{9}{|c|}{ Amphoter. Chondrit } \\
\hline 21 & Dhurmsala & 3,01 & 3,3 & 0,43 & 84 & 0,68 & 32 & 34 \\
\hline
\end{tabular}

Tab. 3. Meßwerte und auf Diffusionsverluste korrigierte Werte der Verhältnisse der spallogenen Edelgase in verschiedenen Mineralkomponenten. ( $\mathrm{M}=$ Metall, $\mathrm{O}=$ Olivin, $\mathrm{P}=$ Pyroxen.) In den Spalten ${ }^{3} \mathrm{He}$ und ${ }^{3} \mathrm{H}$ sind die prozentualen Verluste von ${ }^{3} \mathrm{He}$ und ${ }^{3} \mathrm{H}$ angeführt. Die letzte Spalte enthält die auf Tritiumverluste korrigierten Werte der ${ }^{3} \mathrm{He} /{ }^{21} \mathrm{Ne}-\mathrm{Verhältnisse}$ im Nickeleisen.

Aus Tab. 3 entnehmen wir, daß nur für einige Chondrite im Nickeleisen ${ }^{3} \mathrm{He} /{ }^{21} \mathrm{Ne}$-Werte dieser Größe erreicht werden. Die gemessenen Verhältnisse liegen bis etwa einen Faktor $3 \mathrm{zu}$ tief. Allerdings müssen wir darauf hinweisen, daß gerade die ${ }^{3} \mathrm{He} /{ }^{21} \mathrm{Ne}-$ Verhältnisse im Nickeleisen wegen der sehr geringen ${ }^{21} \mathrm{Ne}-M e n g e n$ mit einem ziemlich großen Fehler behaftet sind. Die Werte in Tab. 1, für ${ }^{21} \mathrm{Ne}_{\mathrm{sp}}$./g gelöst, mußten zweifach korrigiert werden. Das in der $\mathrm{CuCl}_{2}$-Fraktion in Freiheit gesetzte Neon enthält fast immer beträchtliche Mengen von Luftoder Urneon. In dieser Fraktion gehen leider auch immer Silikatspuren - vermutlich Olivin - mit in Lösung. Man könnte daher annehmen, daß das überschüssige Neon aus diesen in Lösung gehenden Oberflächenschichten der Silikate stammt, in welche atmosphärisches Neon hineindiffundiert ist. Gegen diese Annahme spricht die Tatsache, daß frisch gefallene Meteorite wie $\mathrm{Kiel}$ und $\mathrm{Bruderheim}$ in der $\mathrm{CuCl}_{2}$-Fraktion ähnliche Mengen von Überschußneon enthielten als die vor ca. 100 Jahren gefallenen Meteorite $\mathrm{New} \mathrm{C}$ on cord, Parnallee und $\mathrm{Mocs}$. H a y e s Ce n ter, ein Fund, hingegen enthielt reines Spallationsneon. Gerade dieser Meteorit wurde von allen untersuchten Meteoriten am stärksten erhitzt. Dies zeigt sich schon daraus, daß hier die Nickeleisenphase kein radiogenes Helium enthielt. Vor allem der Befund an $\mathrm{H}$ a y e s $\mathrm{C}$ e $\mathrm{n} \mathbf{t}$ e $\mathrm{r}$ zeigt, daß es sich bei dem in der $\mathrm{CuCl}_{2}$-Fraktion der meisten Meteorite gefundenen Neon, das nicht spallogenen Ursprungs ist, um vermutlich Urneon handelt. Die Probe des Bronzit-Chondriten Culli s o $n$ enthält in allen Fraktionen Urneon.

Die Troilitfraktionen enthielten mehrfach große Mengen von Luftneon. Bromdämpfe greifen Hahnfett sehr stark an, so daß es leicht zu starken unkontrollierbaren Erhöhungen des Neonblindwertes kommt. Bei den beträchtlichen Mengen von Überschußneon in der $\mathrm{CuCl}_{2}$ - und Bromphase des BronzitChondriten $\mathrm{R}$ os e City handelt es sich vermutlich um Urneon.

Alle Werte für ${ }^{21} \mathrm{Ne}_{\mathrm{sp}}$./g gelöst in Tab. 1 beziehen sich nur auf die Spallationsanteile. 
Die wenn auch nur sehr kleinen Silikatmengen, die in der $\mathrm{CuCl}_{2}$-Fraktion mitgelöst werden, enthalten dennoch nicht vernachlässigbare Mengen von ${ }^{21} \mathrm{Ne}$. Alle Werte für ${ }^{21} \mathrm{Ne}$ in $\operatorname{der} \mathrm{CuCl}_{2}$-Fraktion, die mit * bezeichnet sind, wurden für diese Anteile korrigiert. Die angegebenen Werte beziehen sich also nur auf das reine Nickeleisen. In einigen Fällen erschien uns eine solche Korrektur nicht gerechtfertigt, und zwar an solchen Meteoriten, in denen große Diffusionsverluste aufgetreten sind. Hier sind wohl die Edelgaskonzentrationen in den äußersten Schichten der Silikatkörner bereits stark vermindert. Allerdings werden beim Pulverisieren der Proben teilweise neue Oberflächen geschaffen.

Trotz dieser Korrekturen sollte das ${ }^{3} \mathrm{He} /{ }^{21} \mathrm{Ne}-\mathrm{Ver}$ hältnis im Nickeleisen im allgemeinen auf ca. $20 \%$ genau sein. Ein noch höherer Fehler gilt nur für einige Meteorite, bei denen die Versuchsbedingungen besonders ungünstig lagen. $\mathrm{Zu}$ diesen Meteoriten gehören Akaba, Parnallee und Dhurmsala.

Die ${ }^{3} \mathrm{He} /{ }^{21} \mathrm{Ne}-$ Verhältnisse im Nickeleisen liegen jedoch gegenüber den zu erwartenden Werten häufig so tief, daß Meßfehler zur Erklärung dieser Diskrepanz nicht ausreichen. Wir vermuten, daß die ${ }^{21} \mathrm{Ne}-$ Konzentration im Nickeleisen teilweise durch aus den Silikaten eingeschossene ${ }^{21} \mathrm{Ne}-K e r n e$ erhöht wurde. Ein ${ }^{21} \mathrm{Ne}-\mathrm{Kern}$, der zuvor ein $\alpha$-Teilchen von $10 \mathrm{MeV}$ emittiert hat, erhält eine Rückstoßenergie von $2 \mathrm{MeV}$. Dieses entspricht im Eisen einer Reichweite von ca. $1 \mu$. Ob dieses eingeschossene ${ }^{21} \mathrm{Ne}$ zur

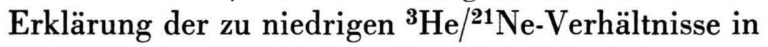
allen Fällen ausreicht, läßt sich schwer abschätzen. Sicher ist, daß die auf diese Weise herrührenden ${ }^{21} \mathrm{Ne}$-Mengen im Nickeleisen nicht vernachlässigbar sind.

\section{Zusammenfassung}

Auf Grund von Edelgasmessungen an einzelnen Mineralkomponenten von Chondriten konnte gezeigt werden:

1. Die Diffusionsverluste von radiogenem als auch von spallogenem Helium im Pyroxen sind teilweise beträchtlich.

2. Der Olivin ist gegenüber Diffusionsverlusten ziemlich unempfindlich.

3. Bei der Mehrzahl der Bronzit-Chondrite mit Verlusten von radiogenem Helium fanden diese erst während der Einwirkung der Höhenstrahlung statt. Der Grund dieser Diffusionsverluste liegt in einer stärkeren Erwärmung auf Grund einer der Sonne näheren Bahn.
4. Bronzit-Chondrite mit leichten Uredelgasen zeigen in den uredelgasfreien hellen Anteilen gleiche relative Verluste von radiogenem und spallogenem Helium. Eine eindeutige Erklärung hierfür ist noch nicht möglich. Es wäre jedoch denkbar, daß es sich um einen Korngrößeneffekt handelt.

5. Unter den Hypersthen-Chondriten sind Verluste von radiogenem Helium im Meteoritenmutterkörper sehr häufig. Fünf von den zehn untersuchten Chondriten dieser Klasse zeigen im Pyroxen einen sehr geringen Gehalt an ${ }^{4} \mathrm{He}_{\mathrm{rad}}$. Aus dem für alle fünf Meteorite recht ähnlichen Heliumgehalt errechnet sich ein Alter von $560 \cdot 10^{6}$ Jahren. Wir können aus unseren Versuchen jedoch nicht entscheiden, ob es sich hierbei um ein echtes Alter handelt.

6. Die großen Variationen des ${ }^{3} \mathrm{He} /{ }^{21} \mathrm{Ne}-$ Verhältnisses, wie sie in Edelgasanalysen an Gesamtproben gefunden wurden, können nicht durch Diffusionsverluste erklärt werden. Variationen um über einen Faktor $4(1,9$ bis 8,2) im Olivin wurden gefunden.

7. Für fast alle untersuchten Meteorite fanden sich im Nickeleisen im Vergleich zum Olivin zu niedrige

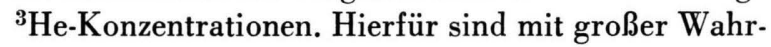
scheinlichkeit Tritiumverluste im Weltraum verantwortlich.

8. Die Tritiumverluste aus dem Nickeleisen gehen

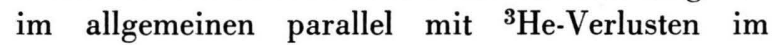
Pyroxen. Es gibt jedoch deutliche Ausnahmen. Eine dieser Ausnahmen ist der uredelgashaltige BronzitChondrit Breits cheid.

9. Die Radioelemente Uran und Thorium sind in Chondriten hauptsächlich im Pyroxen bzw. eventuell in den Feldspäten enthalten.

Für die bereitwillige Überlassung von Meteoritproben danken wir herzlich den Herren Prof. Dr. W. von Engelhardt und Dr. W. Weiskirchner, Tübingen (Tabor), Prof. Dr. R. E. Folinsbee, Edmonton (Bruderheim; vermittelt durch Prof. Dr. H. E. Suess), Prof. Dr. E. Hellner und Dr. W. Schreyer, Kiel (Kiel), Dr. E. P. Henderson, Washington (Rose City und Walters), Prof. Dr. C. W. Conrens und Prof. Dr. S. Koritnig, Göttingen (Parnallee), Dr. C. W. Moore, Temple (Pantar), Dr. E. Olsen, Chicago (Bath). Die Meteorite Akaba, Beddgelert, Breitscheid, Dhurmsala, Mocs, New Concord, Ochansk und Pultusk entstammen der Sammlung F. A. Paneth.

Für ihre Mitarbeit danken wir Fräulein H. BadDENhausen, Fräulein I. Schüler und Fräulein M. Monerjan sowie Herrn B. Spettel.

Der Deutschen Forschungsgemeinschaft sind wir für die leihweise Überlassung von Geräten und dem Bundesministerium für wissenschaftliche Forschung für weitere Sachbeihilfen zu Dank verpflichtet. 